\title{
Scalar magnetic difference inversion applied to UAV-based UXO detection
}

\author{
Kolster, Mick Emil; Døssing, Arne
}

Published in:

Geophysical Journal International

Link to article, DOI:

10.1093/gji/ggaa483

Publication date:

2021

Document Version

Publisher's PDF, also known as Version of record

Link back to DTU Orbit

Citation (APA):

Kolster, M. E., \& Døssing, A. (2021). Scalar magnetic difference inversion applied to UAV-based UXO detection. Geophysical Journal International, 224(1), 468-486. https://doi.org/10.1093/gji/ggaa483

\section{General rights}

Copyright and moral rights for the publications made accessible in the public portal are retained by the authors and/or other copyright owners and it is a condition of accessing publications that users recognise and abide by the legal requirements associated with these rights.

- Users may download and print one copy of any publication from the public portal for the purpose of private study or research.

- You may not further distribute the material or use it for any profit-making activity or commercial gain

- You may freely distribute the URL identifying the publication in the public portal

If you believe that this document breaches copyright please contact us providing details, and we will remove access to the work immediately and investigate your claim. 
Advance Access publication 2020 October 08

GJI Marine Geosciences and Applied Geophysics

\title{
Scalar magnetic difference inversion applied to UAV-based UXO detection
}

\author{
Mick Emil Kolster ${ }^{\circledR}$ and Arne Døssing \\ CMAGTRES, DTU Space, Division of Geomagnetism, The Technical University of Denmark, Kgs. Lyngby,Denmark.E-mail: memkol@space.dtu.dk
}

Accepted 2020 October 6. Received 2020 October 5; in original form 2020 May 11

\begin{abstract}
SUMMAR Y
During scalar magnetic surveys, where the amplitude of the magnetic field is measured, small changes in towed sensor positions can produce complex noise-resembling signals in the data. For well-constructed measurement systems, these signals often contain valuable information, rather than noise, but it can difficult to realize their potential. We present a simple, general approach, which can be used to directly invert data from scalar magnetic surveys, regardless of dynamic or unexpected sensor position variations. The approach generalizes classic alongtrack gradients to an iterative, or recursive, difference, that can be applied irrespective of the amount of magnetic sensors and their positions within a dynamic measurement system, as long as these are known. The computed difference can be inverted directly, providing a versatile method with very little data pre-processing requirements, which we denote as recursive difference inversion. We explain the approach in a general setting, and expand it to provide a complete framework for Unexploded Ordnance (UXO) detection using a pointdipole model. Being an extension of classic along-track gradients, the method retains many of the same properties, which include added robustness to external time-dependent disturbances, and the ability to produce aesthetic visual data representations. In addition, the framework requires neither tie lines, data levelling, nor diurnal corrections. Only light pre-processing actions, namely initial survey trimming and data position calculation, are required. The method is demonstrated on data from a dual sensor system, conventionally referred to as a vertical gradiometer, which is towed from an Unmanned Aerial Vehicle. The system enables collection of high-quality magnetic data in adverse settings, and simultaneously reduces the risk of inadvertent UXO detonations. To enable qualitative testing, we established a UXO detection test facility with several buried UXO, typical to World War II, in a magnetically complex in-land area. Data from the test facility was mainly used to evaluate inversion robustness and depth accuracy of the point-dipole model. Subsequently, we apply the method to real UXO survey data collected for the Hornsea II offshore wind farm project in the United Kingdom. This data set was collected in a coastal setting, and subject to significant sensor position changes during flight due to varying wind conditions over multiple survey days. This makes the raw data set challenging to interpret directly, but it can still be easily and reliably inverted for source locations through recursive difference inversion. In each of the two data sets, we attempt to recover UXO positions using recursive difference inversion on data from both a single sensor, as well as on data from two synchronized sensors, in each case inverting the difference directly for point-dipole model parameters. To seed the inversion, we propose a simple routine for picking out potential targets, based on the choice of a significant peak prominence in the time-series of computed differences. Higher order difference inversion was found to provide robust results in the magnetically complex setting, and the recovered equivalent dipole depths were found to approximate the actual UXO depths well.
\end{abstract}

Key words: Inverse theory; Numerical approximations and analysis; Numerical modelling.

\section{INTRODUCTION}

Scalar magnetic surveys have been performed for over $50 \mathrm{yr}$ using a variety of magnetic measurement systems. Applications include surveying for Unexploded Ordnance (UXO), which pose risks in both current and prior conflict zones, for example, to construction projects in regions 
where old UXO may persist. Common UXO survey methods including towing sensors from surveying ships (e.g. Salem et al. 2005) or mounting them on land vehicles (e.g. Nelson \& McDonald 2001), while niche methods include helicopter mounted arrays (e.g. Gamey et al. 2002). Data processing options are vast, but commonly include filtering, levelling and applications of finite-difference approximated gradients. Unfortunately, conventional UXO survey methods fall short in certain settings, for example, rough or inaccessible terrain and shallow water areas, and can be costly, either monetarily or in terms of risk. Furthermore, conventional data processing strategies, for example, filtering, may also incur a toll on the overall data quality, particularly on the magnetic signatures of smaller UXO. As such, there exists a need for more versatility within UXO surveying, ideally enabling challenging regions to be surveyed, without necessitating disruptive data transformations.

Recent developments within Unmanned Aerial Vehicles (UAVs) have resulted in various UAV-borne magnetic surveying systems (e.g. Stoll 2013). UAV systems enable operation in difficult areas while retaining low risk levels and operational costs, but also introduce new challenges: the magnetic field of most UAVs is highly dynamic and disturbing; the take-off weight is limited, often resulting in the inclusion of only a single scalar field magnetometer; and the sensor attachment strategy is not straightforward. Rigidly attached sensors are often closer to the UAV, sharing changes in attitude and introducing noise from the UAV, while systems towed further away (e.g. in strings, as in Cherkasov et al. 2016; Tuck et al. 2019), reduce magnetic noise (e.g. Parshin et al. 2018), but enable sensor position variations, for example, through swinging effects. UAV-induced errors require noise compensation (e.g. Cherkasov et al. 2016; Tuck et al. 2019), while towed sensors locations can be measured with dedicated instruments as in magnetometer birds (e.g. Wyckoff 1948; Giret 1965), or approximated from the UAV position, adding a position-induced bias. Walter et al. (2019a) found that swinging outside the measurable disturbance of the UAV introduced variations of $\leq 0.5 \mathrm{nT}$ in a geological setting. This error is significant for UXO surveying, and even more so if utilizing multiple sensors, as the bias may vary between them. Walter et al. (2019a) applied a low-pass filter to the swing-affected geological survey, which cannot be replicated for UXO surveys, due to potential bandwidth overlaps with targets. As such, to circumvent filtering, sensors should be both accurately positioned, and placed far from the UAV to eliminate noise. However, an increase of the sensor-to-UAV distance may result in larger perturbations of the sensor positions, due to the dynamic movement of the UAV. For multisensor approaches, the effect of increased perturbation is twofold, as not only sensor positions, but also the sensor alignment axis (axis through a pair of sensors) may vary in the local frame of reference, for example, sensors which are vertically aligned when at rest may not be vertically aligned in motion. Thus, in the case of large perturbations, some conventional methods may be troublesome to apply without imposing undesirable transformations, or simply inapplicable, e.g. if finite-difference approximations to the gradient are poor, which may very well be the case as both source distance and its signature spectrum are generally not known a priori. Fortunately, many of the aforementioned challenges can be alleviated using a generalized approach, which we denote recursive difference inversion (RDI).

RDI poses a simple approach to source model inversion directly on measured or calculated differences. The approach is valid irrespective of sensor amount and configuration, and circumvents assumptions inherent to finite-difference approximations, instead only requiring a small enough data spacing to reliably capture variations in source signatures. In the following, we explain the method and demonstrate solution of the inherently nonlinear inverse problem of determining model parameters (herein source location), with a specially designed Fletchermodified Levenberg-Marquardt (LM) algorithm (see e.g. Fletcher 1971; Levenberg 1944; Marquardt 1963). While applicable to single sensor data uncorrected for external disturbances, additional resolution and robustness to external noise, including diurnal variations, is gained when utilizing two or more synchronized sensors (Cowan et al. 1995). We demonstrate the method on data from a novel UAVtowed magnetic measurement system, which combines dedicated positioning sensors with two synchronized scalar magnetometers. We apply RDI individually on both single and dual sensor data, in each of two distinctly different scenarios: a UXO detection test facility in a magnetically complex in-land area, where several known UXO are buried, and a UXO survey related to construction of the Hornsea II Offshore Wind Farm (UK), in a combined intertidal and shallow-water coastal setting. Data from the test facility are used to demonstrate important properties of the method, while the coastal survey demonstrates application to a conventionally inaccessible area within offshore construction.

\section{METHOD}

Scalar magnetometers measure the magnetic field amplitude, also referred to as the total or scalar magnetic field, in units of Tesla, that is, the Euclidean two-norm of the magnetic vector field. For most practical scenarios, including magnetic surveying for UXO, the relation between data and sources is nonlinear. To derive a set of equations suitable for source inversion, we first expand the measured field amplitude $\mathrm{d}(\mathbf{x}, t)$ as a nonlinear combination of three entities: the core field $\mathbf{B}_{\text {core }}(\mathbf{x}, t)$, which stems from complex dynamo motions in the Earth's outer core; the external field $\mathbf{B}_{\text {ext }}(\mathbf{x}, t)$, due to geomagnetic sources outside the Earth's surface, including any temporally varying anthropogenic signals; and the anomaly field $\mathbf{B}_{a}(\mathbf{x}, t)$, due to a combination of induced and permanent magnetization contained in the Earth's crust, including temporally quasi-invariant supracrustal objects, such as UXO, as well as any sensor noise present in the measurements. This enables the scalar field data $\mathrm{d}(\mathbf{x}, t)$ measured at the sensor head of the magnetometer to be described through eq. (1).

$\mathrm{d}(\mathbf{x}, t)=\left|\mathbf{B}_{\text {core }}(\mathbf{x}, t)+\mathbf{B}_{a}(\mathbf{x}, t)+\mathbf{B}_{\text {ext }}(\mathbf{x}, t)\right|_{2}$

Generally, $\left|\mathbf{B}_{\text {core }}+\mathbf{B}_{a}\right|_{2} \gg\left|\mathbf{B}_{\text {ext }}\right|_{2}$, enabling reduction to eq. (2) through the triangle inequality.

$\mathrm{d}(\mathbf{x}, t) \approx\left|\mathbf{B}_{\text {core }}(\mathbf{x}, t)+\mathbf{B}_{a}(\mathbf{x}, t)\right|_{2}+\left|\mathbf{B}_{\text {ext }}(\mathbf{x}, t)\right|_{2}$ 
A simple but effective approach is to now expand eq. (2) as a Taylor series anchored at $\mathrm{B}_{a}=0$, based on the notion that $\mathrm{B}_{\text {core }} \gg \mathrm{B}_{a}$. For many purposes, including UXO detection, it is generally sufficient to keep only the zeroth- and first-order terms, yielding eq. (3).

$\mathrm{d}(\mathbf{x}, t) \approx \mathrm{B}_{\text {core }}(\mathbf{x}, t)+\mathrm{B}_{\mathrm{ext}}(\mathbf{x}, t)+\hat{\mathbf{B}}_{\text {core }}(\mathbf{x}, t) \cdot \mathbf{B}_{a}(\mathbf{x}, t)$

where $\hat{\mathbf{B}}_{\text {core }}$ is the unit direction of $\mathbf{B}_{\text {core }}$. While each entity in eq. (3) is in essence a function of both position $\mathbf{x} \in \mathfrak{R}^{3}$ and time $t$, several simplifications are available, and in some cases some dependencies can often be assumed negligible: temporal variation of the core and crustal fields may be assumed temporally independent if their timescale of variation, which is generally on the order of years (Olsen et al. 2014), is significantly larger than the magnetic survey duration. Likewise, the core field direction and external magnetic field may be assumed independent of position for magnetic surveys of limited spatial extent. Via these assumptions, the calculated first difference (difference between two samples of the same sensor) can be obtained through eq. (4).

$\Delta^{1} \mathrm{~d}\left(\mathbf{x}_{\alpha}, \mathbf{x}_{\beta}, t_{\alpha}\right) \approx \Delta^{1} \mathrm{~B}_{\text {core }}\left(\mathbf{x}_{\alpha}, \mathbf{x}_{\beta}, t_{\alpha}\right)+\Delta^{1} \mathrm{~B}_{\text {ext }}\left(t_{\alpha}, t_{\beta}\right)+\hat{\mathbf{B}}_{\text {core }}\left(\mathbf{x}_{\alpha}\right) \cdot \Delta^{1} \mathbf{B}_{a}\left(\mathbf{x}_{\alpha}, \mathbf{x}_{\beta}, t_{\alpha}\right)$

where $\Delta \mathrm{B}_{\text {ext }}\left(t_{\alpha}, t_{\beta}\right)$ denotes the external variation between measurement times $t_{\alpha}$ and $t_{\beta}$. Eq. (4) is generally reliable if $\mid \hat{\mathbf{B}}_{\text {core }}\left(\mathbf{x}_{\alpha}\right)$. $\Delta \mathbf{B}_{a}\left(\mathbf{x}_{2}, \mathbf{x}_{1}, t_{\alpha}\right)|\gg| \Delta \mathrm{B}_{\text {ext }}\left(t_{\alpha}, t_{\beta}\right) \mid$, which is more likely if the time difference $\left|t_{\alpha}-t_{\beta}\right|$ is minimized, that is, by using temporally adjacent data points, but this cannot generally be guaranteed a priori. Eq. (4) can highlight high-frequency variations, provided that the sample spacing is small enough to accurately recover them without being drowned out by noise. Eq. (4) also subdivides external signal contributions between each set of data points, which is particularly useful for inversion purposes, as the error will be significantly less structured in the spatial domain (provided geomagnetically quiet conditions). As such, eq. (4) reduces the amount of available information compared to eq. (3), but this is arguably mainly positive for well-spaced sample spacings, that is, small enough to retain high frequencies, while large enough to retain a distinguishable anomaly signal amongst any present noise signals.

The inclusion of at least two synchronized sensors removes the dependence of time-varying sources outside their immediate vicinity, nullifying the need for the first stated assumption. For simplicity, we shall now assume that the core field direction and external field amplitude are negligible across magnetic sensors, provided that they stay in the immediate vicinity of each other. In this setting, the difference between the sensor at position $\mathbf{x}_{\alpha}$ and the sensor at position $\mathbf{x}_{\beta}$, at any given time $t_{\gamma}$, can be expressed through eq. (5).

$\mathrm{d}\left(\mathbf{x}_{\alpha}, t_{\gamma}\right)-\mathrm{d}\left(\mathbf{x}_{\beta}, t_{\gamma}\right) \approx \mathrm{B}_{\text {core }}\left(\mathbf{x}_{\alpha}, t_{\gamma}\right)-\mathrm{B}_{\text {core }}\left(\mathbf{x}_{\beta}, t_{\gamma}\right)+\hat{\mathbf{B}}_{\text {core }}\left(\mathbf{x}_{\alpha}, t_{\gamma}\right) \cdot\left(\mathbf{B}_{a}\left(\mathbf{x}_{\alpha}, t_{\gamma}\right)-\mathbf{B}_{a}\left(\mathbf{x}_{\beta}, t_{\gamma}\right)\right)$

Using $\Delta^{1}$ to denote the first difference between data measured at positions $\mathbf{x}_{\alpha}$ and $\mathbf{x}_{\beta}$, we arrive at eq. (6).

$\Delta^{1} \mathrm{~d}\left(\mathbf{x}_{\alpha}, \mathbf{x}_{\beta}, t_{\gamma}\right) \approx \Delta^{1} \mathrm{~B}_{\text {core }}\left(\mathbf{x}_{\alpha}, \mathbf{x}_{\beta}, t_{\gamma}\right)+\hat{\mathbf{B}}_{\text {core }}\left(\mathbf{x}_{\alpha}, t_{\gamma}\right) \cdot \Delta^{1} \mathbf{B}_{a}\left(\mathbf{x}_{\alpha}, \mathbf{x}_{\beta}, t_{\gamma}\right)$

where the assumption of temporal invariance in the core and anomaly fields, for the duration of magnetic data collection, would enable all $t_{\gamma}$ dependencies to be neglected.

The core field entities $\mathbf{B}_{\text {core }}$ and $\Delta^{1} B_{\text {core }}$ in eqs (4) and (6) can be approximated through a core field model (e.g. Finlay et al. 2016). For smaller surveys, it may also be reasonable to assume a general direction of $\hat{\mathbf{B}}_{\text {core }}$ for the entire survey, and that $\Delta^{1} \mathrm{~B}_{\text {core }}$ is mainly a function of altitude.

A possible continuation to eq. (6) is to compute approximate gradients through one of the multiple available methods (e.g. Nabighian 1984; Wang et al. 2008), and derive higher order data products such as the analytic signal (Roest et al. 1992), or estimate the source position through Euler deconvolution (Thompson 1982). Application of RDI, however, only requires computation of differences in measured data. Using eq. (6), we can define higher order (recursive) differences, for distinct sensor positions $\mathbf{x}_{\alpha m}$ and $\mathbf{x}_{\beta m}$, where $m$ denotes a unique combination of two positions where data have been captured (as also explained in Fig. 1). The second-order difference is thus defined by eq. (7).

$\Delta^{2} \mathrm{~d}\left(\mathbf{x}_{\alpha 1}, \mathbf{x}_{\beta 1}, \mathbf{x}_{\alpha 2}, \mathbf{x}_{\beta 2}\right)=\Delta^{1} \mathrm{~d}\left(\mathbf{x}_{\alpha 1}, \mathbf{x}_{\beta 1}\right)-\Delta^{1} \mathrm{~d}\left(\mathbf{x}_{\alpha 2}, \mathbf{x}_{\beta 2}\right)$

where we consider the underlying dependence on time implicit, and therefore have discontinued repeating the time variable. We stress that the time dependence is ever present, requiring compensation unless reasonable to assume negligible.

In general, differences of order $k \geq 2$ can defined by the recurrence in eq. (8).

$\Delta^{k} \mathrm{~d}\left(\mathbf{x}_{\alpha 1}, \mathbf{x}_{\beta 1}, \ldots, \mathbf{x}_{\alpha N}, \mathbf{x}_{\beta N}\right)=\Delta^{k-1} \mathrm{~d}\left(\mathbf{x}_{\alpha 1}, \mathbf{x}_{\beta 1}, . ., \mathbf{x}_{\alpha M}, \mathbf{x}_{\beta M}\right)-\Delta^{k-1} \mathrm{~d}\left(\mathbf{x}_{\alpha M+1}, \mathbf{x}_{\beta M+1}, \ldots, \mathbf{x}_{\alpha N}, \mathbf{x}_{\beta N}\right)$

where it follows naturally that $M=2^{k-2}$ and $N=2^{k-1}$, for the $k^{\prime}$ th difference.

We have kept eq. (8) as general as possible, and good choices of input positions $\left(\mathbf{x}_{\alpha 1}, \mathbf{x}_{\beta 1}, \ldots, \mathbf{x}_{\alpha N}, \mathbf{x}_{\beta N}\right)$ in eq. (8) will depend on the objective, for example, pairings of very distant data points will be unsuitable for UXO detection. Random parings of unordered data may also result in accidental loss of information through, for example, increased effective sample spacing or difference order reduction, due to trivialities in the difference calculation process. Perhaps the most natural approach is to use chained neighbouring data points, that is, some variant of classic forward or backwards differences, ordering the data such that $m$ increases with distance traversed along the survey path, and setting $\mathbf{x}_{\beta m}=\mathbf{x}_{\alpha m-1}$ in the above relations. However, classic forward or backwards recurring differences cannot strictly describe the method in the multiple sensor case, due to the two individual dimensions of differences: those computed across multiple (ideally synchronized) sensors, as in eq. (6), and those derived from movement, as in eq. (4). Furthermore, there may be cases where it is less ideal to constrain the difference calculation to adjacent data points, that is, for very dense data spacings, where the dynamic range may be very small or completely enveloped 
Chained single sensor differences

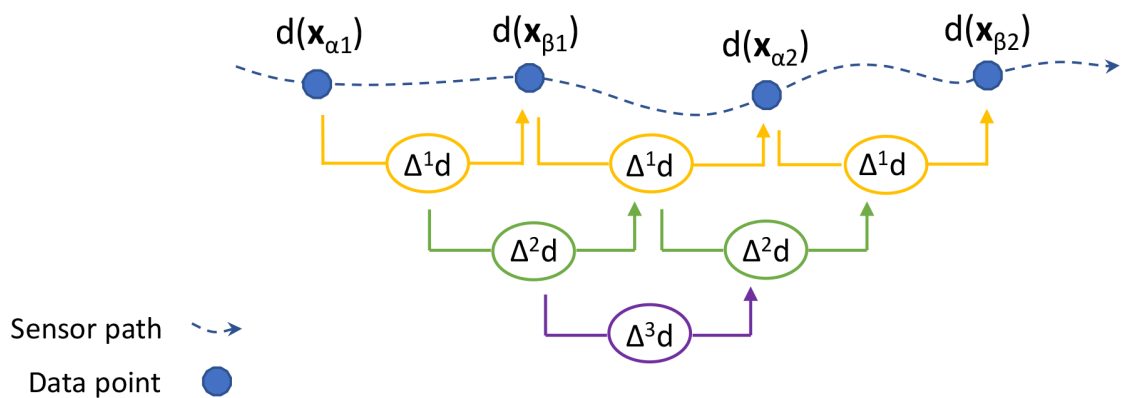

\section{Chained dual sensor differences}

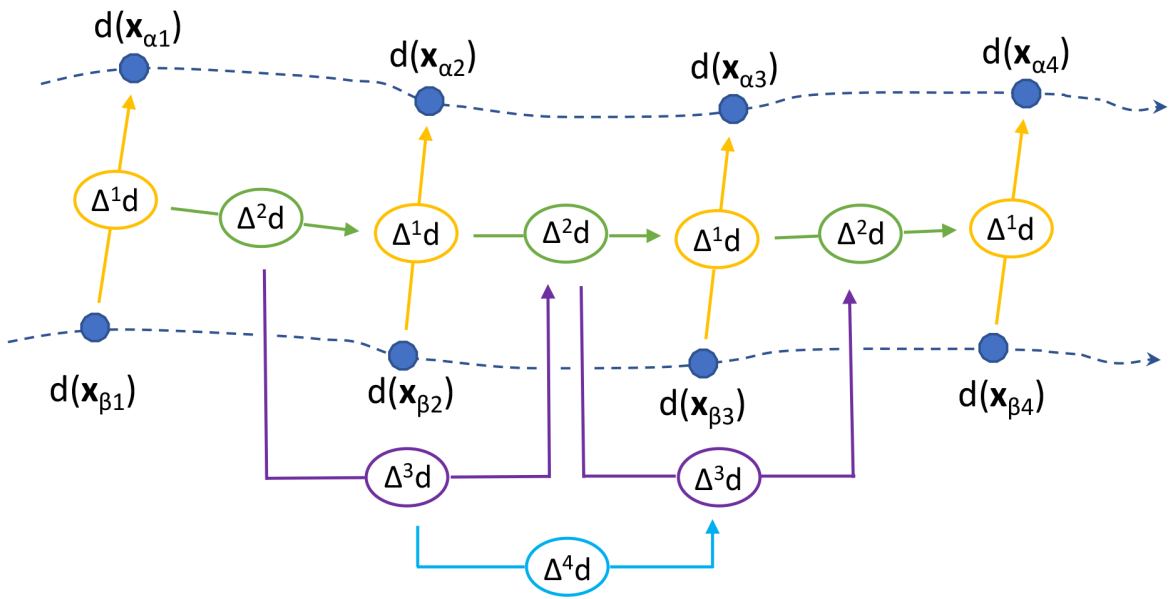

Figure 1. Example of the chained difference calculation approach applied in this study. Chained differences are similar to along track gradients, but do not depend explicitly on the intradata distances, nor do they need to adhere to a specific designated direction.

by the noise floor. While not necessary to downsample the data in such cases, it may be prudent to compute the differences across larger spatial distances than the sample spacing between adjacent data points. This is particularly relevant when considering recent developments within UAV-ready scalar magnetic sensors, which have achieved sampling rates on the order of $10^{2}-10^{3} \mathrm{~Hz}$ (e.g. the Geometrics MFAM).

Successful implementation of RDI requires only a few steps. Eqs (6) and (8) provide the general framework for inverting scalar magnetic field data for a source model able to predict the anomaly field, through a specific set of variables (which would generally include the source location along with other source parameters). Defining the unknown model parameters $\mathbf{p}$, and function $\mathcal{F}$, which together predict the synthesized data $\mathbf{s}$ placed at distinct positions $\mathbf{x}_{\alpha m}$ and $\mathbf{x}_{\beta m}$, the modelled (or model-predicted) first-order difference can be stated as eq. (9).

$\Delta^{1} \mathrm{~s}\left(\mathbf{x}_{\alpha m}, \mathbf{x}_{\beta m}, \mathbf{p}\right)=\mathrm{s}\left(\mathbf{x}_{\alpha m}, \mathbf{p}\right)-\mathrm{s}\left(\mathbf{x}_{\beta m}, \mathbf{p}\right)=\mathcal{F}\left(\mathbf{x}_{\alpha m}, \mathbf{p}\right)-\mathcal{F}\left(\mathbf{x}_{\beta m}, \mathbf{p}\right)$

while higher order differences are obtained analogous to the measured difference, through the recurrence relation

$\Delta^{k} \mathrm{~s}\left(\mathbf{x}_{\alpha 1}, \mathbf{x}_{\beta 1}, \ldots, \mathbf{x}_{\alpha N}, \mathbf{x}_{\beta N}\right)=\Delta^{k-1} \mathrm{~s}\left(\mathbf{x}_{\alpha 1}, \mathbf{x}_{\beta 1}, . ., \mathbf{x}_{\alpha M}, \mathbf{x}_{\beta M}\right)-\Delta^{k-1} \mathrm{~s}\left(\mathbf{x}_{\alpha M+1}, \mathbf{x}_{\beta M+1}, . ., \mathbf{x}_{\alpha N}, \mathbf{x}_{\beta N}\right)$.

For scalar magnetic data, inversion for $\mathbf{p}$ through eq. (6) is generally a nonlinear problem. We here employ a variation of the LM algorithm (Levenberg 1944; Marquardt 1963) with individual dampening weights as proposed by Fletcher (1971). However, it should be noted that the general method is not exclusive to this approach; in principle any nonlinear solver can be used. The LM algorithm does not guarantee convergence to the global minimum, and requires an initial guess of the unknown model parameters. Such guesses can be obtained in various ways; through peaks in the measured data or derived products, through Euler deconvolution, or otherwise.

The LM algorithm relies on the Jacobian matrix of partial derivatives with respect to the model parameters. For inversion on the first measured difference, the Jacobian row elements can be computed through eq. (11).

$\left[\Delta^{1} \mathbf{J}\left(\mathbf{x}_{\alpha m}, \mathbf{x}_{\beta m}, \mathbf{p}\right)\right]_{j}=\frac{\partial}{\partial \mathrm{p}_{j}}\left(\mathcal{F}\left(\mathbf{x}_{\alpha m}, \mathbf{p}\right)-\mathcal{F}\left(\mathbf{x}_{\beta m}, \mathbf{p}\right)\right)=\frac{\partial}{\partial \mathrm{p}_{j}} \mathcal{F}\left(\mathbf{x}_{\alpha m}, \mathbf{p}\right)-\frac{\partial}{\partial \mathrm{p}_{j}} \mathcal{F}\left(\mathbf{x}_{\beta m}, \mathbf{p}\right)$

while higher order Jacobian row elements are obtained through the corresponding recurrence relation

$\left[\Delta^{k} \mathbf{J}\left(\mathbf{x}_{\alpha 1}, \mathbf{x}_{\beta 1}, \ldots, \mathbf{x}_{\alpha N}, \mathbf{x}_{\beta N}\right)\right]_{j}=\left[\Delta^{k-1} \mathbf{J}\left(\mathbf{x}_{\alpha 1}, \mathbf{x}_{\beta 1}, . ., \mathbf{x}_{\alpha M}, \mathbf{x}_{\beta M}\right)\right]_{j}-\left[\Delta^{k-1} \mathbf{J}\left(\mathbf{x}_{\alpha M+1}, \mathbf{x}_{\beta M+1}, . ., \mathbf{x}_{\alpha N}, \mathbf{x}_{\beta N}\right)\right]_{j}$. 
Compiling all positions $\mathbf{x}_{\alpha 1}, \ldots, \mathbf{x}_{\alpha N}$ in $\mathbf{X}_{\alpha}$ and $\mathbf{x}_{\beta 1}, \ldots, \mathbf{x}_{\beta N}$ in $\mathbf{X}_{\beta}$, respectively, the compiled measured and modelled difference vectors, $\Delta^{k} \mathbf{d}$ and $\Delta^{k} \mathbf{s}$, with Jacobian matrix $\Delta^{k} \mathbf{J}$, can be described as

$$
\begin{aligned}
\Delta^{k} \mathbf{d} & =\Delta^{k} \mathbf{d}\left(\mathbf{X}_{\alpha}, \mathbf{X}_{\beta}\right) \\
\Delta^{k} \mathbf{s} & =\Delta^{k} \mathbf{s}\left(\mathbf{X}_{\alpha}, \mathbf{X}_{\beta}, \mathbf{p}_{n}\right) \\
\Delta^{k} \mathbf{J} & =\Delta^{k} \mathbf{J}\left(\mathbf{X}_{\alpha}, \mathbf{X}_{\beta}, \mathbf{p}_{n}\right)
\end{aligned}
$$

which results in the Fletcher-modified LM system seen in eq. (13).

$$
\left(\left(\Delta^{k} \mathbf{J}\right)^{\mathrm{T}}\left(\Delta^{k} \mathbf{J}\right)+\lambda \operatorname{diag}\left(\left(\Delta^{k} \mathbf{J}\right)^{\mathrm{T}}\left(\Delta^{k} \mathbf{J}\right)\right)\right) \delta \mathbf{p}_{n}=\left(\Delta^{k} \mathbf{J}\right)^{\mathrm{T}}\left(\Delta^{k} \mathbf{d}-\Delta^{k} \mathbf{s}\right)
$$

where superscript $\mathrm{T}$ denotes transposition and diag is the diagonal element operator. Eq. (13) must be solved iteratively. Starting from an initial guess of the unknown parameters, $\mathbf{p}_{0}$, we iteratively compute the parameter guess update $\delta \mathbf{p}$ from the modelling error obtained in the previous step, updating $\mathbf{p}_{n+1}=\mathbf{p}_{n}+\delta \mathbf{p}_{n}$ after each successful ( $n$ 'th) iteration, which occurs if, for example, the two-norm of the modelling error $|\Delta \mathbf{d}-\Delta \mathbf{s}|_{2}$ has decreased compared to its previous iteration. Upon each successful iteration, the dynamic dampening parameter $\lambda$ is decreased. In the case of an unsuccessful iteration, $\lambda$ is iteratively increased until a successful iteration can be performed. In either case, the inversion is terminated after reaching a set convergence criteria (e.g. the solution does not change significantly), or the maximum allowed amount of iterations is reached (to counter the possibility of infinite loops). A handy attribute of the differencing method (which is not limited to the LM algorithm) is that existing routines for source inversion with single magnetometer systems can be utilized for the differencing method as well, at very little extra implementation cost. The method can thus serve as a versatile, general framework for magnetic source inversion, being essentially independent of sensor amount and positions, and not strictly adhering to any specific direction or directions. This enables RDI to be performed with minimal pre-processing, as solely the individual sensor positions must be known prior to inversion, and alleviates some of the challenges with magnetometer swing in UXO surveying, as we shall see in the following.

\section{APPLICATION FOR UXO SURVEYING}

A few steps remain for application of RDI to UXO survey data. We require a source model, to define the parameters $\mathbf{p}$, and a strategy for determining the corresponding initial guesses $\mathbf{p}_{0}$. RDI in general poses no limitations on the choice of either, but in order to successfully recover targets, the model should generally be able to approximate the source signatures, while the initial parameter guesses should be close enough to the actual target positions to enable convergence through eq. (13). As explained in the following, we choose to model the targets as point-dipoles model. For the initial target selection algorithm, we impose the requirement of having at least two prominent peaks, one positive and one negative, adjacent to each other in order to consider a variation in the difference as a potential target. It should be noted that scalar magnetic differences generally introduce one additional peak per anomaly than single-sensor magnetic amplitude data, and that peaks are evaluated along the flight track, that is, anomalies that are captured on multiple flight lines generally encompass several peaks per line. As such, we consider the two-peak criteria to be very loose.

\subsection{The point-dipole model}

For detection purposes with magnetic sensors (i.e. approximating the location of shallow sources), it is generally sufficient to model UXO as point dipoles (Butler et al. 2001), and we employ this in the following. Multiple studies have applied the point-dipole model for detection (e.g. McFee \& Das 1981; Gamey et al. 2002; Sanchez et al. 2008; Davis et al. 2010), but not, to our knowledge, for direct inversion with raw measured recursive differences. The point-dipole equation can be easily derived from the multipole expansion of the magnetic potential (e.g. Panofsky \& Phillips 1955):

$\mathbf{B}_{\mathrm{dip}}(\mathbf{r}, \mathbf{m})=\frac{\mu_{0}}{4 \pi}\left(\frac{(\mathbf{r} \cdot \mathbf{m}) \mathbf{r}}{|\mathbf{r}|^{5}}-\frac{\mathbf{m}}{|\mathbf{r}|^{3}}\right)$

where $\mathbf{B}_{\text {dip }}$ is the magnetic vector field of the dipole, $\mu_{0}$ is the magnetic permeability of a vacuum, $\mathbf{m}$ is the dipole moment and $\mathbf{r}=\mathbf{x}-\mathbf{x}_{0}$ is the vector from the source coordinate $\mathbf{x}_{0}$ to the data coordinate $\mathbf{x}$ in a Cartesian coordinate system. The nonlinear system of equations to be solved for source parameters $\mathbf{m}$ and $\mathbf{x}_{0}$ is then obtained by inserting eq. (14) into eq. (6).

\subsection{Initial parameter guess}

In order to invert the data for equivalent point dipoles (i.e. the part of the target signatures that can be modelled with a point dipole), we first devise a strategy for obtaining the initial parameter guess $\mathbf{p}_{0}=\left[\mathbf{x}_{0}, \mathbf{m}_{0}\right]^{\mathrm{T}}$. We evaluate this method against the test facility data, and subsequently employ it to the coastal survey.

The horizontal component of the position guess $\mathbf{x}_{0}$ is obtained from peaks in the data time-series with prominences above one data standard deviation. The underlying assumption is that UXO contain ferrous material, resulting in enhanced signal amplitudes relative to the background signal, and that the cost of detection may increase significantly for lower values (which we also test in the following). For each potential target, we require at least two peaks in the difference data (herein at least one positive and one negative), within a radius of two line 
Table 1. Overview of survey and sensor parameters. As described in the text, the sensor absolute accuracy and orientation error are expected to have less influence on the final result. The perhaps most important parameter is the sensor sensitivity, that is, the minimum change in the magnetic field that can be detected by the sensors.

\begin{tabular}{lc}
\hline Parameter & Value \\
\hline UAV ground speed & $5 \mathrm{~m} \mathrm{~s}^{-1}$ \\
Along-track sample distance & $0.25 \mathrm{~m}$ \\
Sensor sampling rate & $20 \mathrm{~Hz}$ \\
Sensor sensitivity & $3 \mathrm{pT}($ at $20 \mathrm{~Hz})$ \\
Absolute accuracy & $\pm 100 \mathrm{pT}$ \\
Sensor resolution & $0.1 \mathrm{pT}$ \\
Sensor orientation error & $\pm 50 \mathrm{pT}$ \\
\hline
\end{tabular}

spacings, that is, the peaks can be on neighbouring lines. Should this result in multiple potential targets in close proximity, we recursively average points within that same radius. The vertical coordinate guess is less straightforward, and we therefore evaluate the impact of bad initial vertical coordinate guesses using the UXO test facility data.

The initial dipole moment guesses $\mathbf{m}_{0}$ are obtained as the unit magnetic dipole moment in the direction of the core field. This approach poses two main challenges: the direction of the dipole moment may not be purely induced, that is, the direction may be incorrect, and UXO exist in numerous shapes, with unknown magnetizations, that is, the amplitude of the dipole moment may also not be correct. We therefore already suspect that all $\mathbf{m}_{0}$ guesses are poor, which may derail the iterative solution of eq. (13) during the first few iterations. To counter this, we initiate the inversion with an increased dampening parameter value, setting $\lambda=10^{3}$, while enforcing a limited update rate $v=\sqrt{2}$. This slows down the inversion process, but diminishes the impact of the (suspected bad) Hessian approximation in eq. (13), forcing the algorithm away from Gauss-Newton behaviour toward gradient descent in the first iterations. The final consideration is the data used in the equivalent dipole calculation. Here, we choose to demean a subset of the data within a fixed radius, and compute the equivalent point dipole using that data. We denote this as the data inclusion radius, for which variations are also investigated using the test facility data, before application in the coastal survey.

\subsection{Data measurement setup, uncertainties and pre-processing}

The data for this study were collected by towing a payload with two GSMP-35U scalar-field magnetometers and accompanying navigation sensors several metres beneath a DJI Wind 4 UAV. The magnetometers are vertically aligned when the system is at rest, that is, the sensors comprise what is known as a vertical gradiometer in conventional terms. However, while the sensors are rigidly attached to each other within the payload, the payload itself is non-rigidly attached to the UAV, thus retaining a large degree of rotational and movement freedom. As such, the sensor positions are kept fixed with respect to each other, but the axis along which they are aligned may deviate from the vertical during flight, for example, due to UAV accelerations and wind effects.

The distance between sensors was $80 \mathrm{~cm}$ at the test facility, and $85 \mathrm{~cm}$ at the coastal survey. The payload positioning system was placed $1.20 \mathrm{~m}$ and 1.15 above the upper sensor at the test facility and coastal survey, respectively. In order to minimize magnetic interference from the Wind 4 platform, Walter et al. (2019a) propose a distance of $\sim 5 \mathrm{~m}$ between UAV and sensor. The setup employed to collect data for this study kept sensors at $\sim 4.5$ and $\sim 5.3 \mathrm{~m}$ below the UAV. Additional survey parameters and sensor specifications are provided in Table 1 .

The position of the UAV was obtained through a D-RTK system (with an accuracy on the order of centimetres, relative to the stationary RTK base station), while dedicated navigation sensors on the payload system itself provided independent payload position and attitude. Since the payload positioning system (a GNSS setup utilizing corrections from the European Geostationary Navigation Overlay Service) is unable to sufficiently constrain the vertical data coordinate, we include D-RTK data from the UAV to constrain the vertical data positions. This is possible because the ray through the two sensors almost always intersects with the UAV. By assuming that this is always true, we can estimate the payload altitude relative to the UAV D-RTK position through the fixed dimensions of the towing and payload system, and the payload attitude. To obtain absolute altitudes, we reference the D-RTK position of the UAV to the Danish National elevation model (DK-DEM, with a cell size of $0.4 \mathrm{~m}$ ) at the test facility, which was also used to drape the survey over the terrain. For flights over the sea or at the coast, the vertical datum is specified relative to the local sea surface. An overview of the error sources for each of the entities contributing to the final data positions, as well as the total absolute positioning uncertainties of the data, are shown in Table 2. The total absolute errors are defined as the error with respect to an external datum. It should be noted that the positioning error between temporally adjacent samples, that is, the relative positioning error of neighbouring samples, will be significantly less than the absolute errors. This is mainly due to the small time span between samples, which limits the maximum possible change in, for example, navigation satellite constellations and atmospheric composition (for details on the general principles of satellite based positioning, see e.g. Misra \& Enge 2006). A coarse estimate of this error was obtained by considering the drift of the positioning estimate when utilizing three data snippets of $\sim 2$ min each, one each in the beginning and end of the survey, and one in the middle. From these data snippets, the mean horizontal drift between samples was found to be $\sim 1 \mathrm{~mm}$ in the horizontal direction. Due to the more complex approach used to determine the sensor altitudes, evaluation of the 
Table 2. Contributions to the total absolute positioning uncertainties of the magnetic data. The maximum uncertainty estimate is obtained from simple addition of relevant sources, and is a measured of the expected maximum deviation from an external datum. The vertical attitude uncertainty is significantly higher than the horizontal payload attitude because it is referenced to the UAV D-RTK position (resultant attitude-based positioning uncertainties are proportional to the distance to the reference point). The attitude-based uncertainty also differs between sensors, as well as on the specific payload angle; in this case we provide the upper uncertainty bound (corresponding to the largest possible uncertainty on the lower sensor position), rounded up to nearest centimetre. The relative positioning error (expected error of one data point relative to the previous one) was estimated from a static test to be $<1 \mathrm{~mm}$ in the horizontal direction.

\begin{tabular}{lcc}
\hline Source of uncertainty & Horizontal uncertainty & Vertical uncertainty \\
\hline Payload position (GNSS w. & $\leq 0.60 \mathrm{~m}$ & - \\
EGNOS) & - & $2 \mathrm{~cm}+1 \mathrm{ppm}$ \\
UAV D-RTK & $\leq 5 \mathrm{~cm}$ & $\leq 10 \mathrm{~cm}$ \\
Payload attitude & - & $0.05 \mathrm{~m}$ \\
DK-DEM Elevation model & $\sim 0.65 \mathrm{~cm}$ & $\sim 0.17 \mathrm{~cm}$ \\
Maximum uncertainty estimate &
\end{tabular}

relative altitude error is not as straightforward, however we expect this to be similarly small, since the rms position drift due to D-RTK is specified at $0.3 \mathrm{~m} \mathrm{~s}^{-1}(0.75 \mathrm{~mm}$ per sample spacing of $25 \mathrm{~cm})$, while the extracted data snippets revealed a mean altitude coordinate drift of $\sim 2 \mathrm{~mm}$

During surveys, adjacent flight lines were flown in opposite directions, but the yaw of the UAV was kept constant (a unique ability of helicopter and multirotor style UAVs). The main advantage of yaw-fixation in this study is that the slight $( \pm 50 \mathrm{pT})$ sensor orientation error, that is, the maximum possible error due to changes in the angle between sensor and magnetic field, was minimized. Yaw-fixation can provide significant advantages when utilizing the specific sensors employed here (see e.g. Walter et al. 2019b), however the benefits of yaw-fixation in this study are slightly limited, due to longitudinal alignment of the sensors. We further note that for data products involving at least one along-track difference, the effect of sensor orientation errors on individual data points will be small, due to the sampling rate being significantly faster than the rate of change of the payload attitude during individual lines. The absolute accuracy, that is, the potential error due to a fixed offset of the sensor, is also expected to be of lesser importance, since the sensors are rigidly attached, and because differences are inherently relative measurements.

Data pre-processing consist solely of data trimming (cutting data during turns) and the described sensor position calculation. Trimming involves removing data collected during UAV turns, landings and take-offs, and the removal of any unlocked measurements, which occurs if the measured field coincides with the sensor dead zones.

\section{RESULTS}

Data from UXO surveys in two distinct settings are presented: (i) at a UXO detection test facility in Denmark with complex magnetic background, where 12 known UXO are buried and (ii) in a real UXO survey scenario in relation to the construction of an offshore wind farm on the east coast of Great Britain. The test facility provides a complex magnetic background, which includes many non-UXO targets, while the coastal survey introduces larger sensor-source separation, unknown seabed elevation, and strong, varying winds. We construct a semi-automatic routine to acquire the initial positions guesses of potential targets, and invert for a point-dipole model at each position guess, using each of the first few differences.

\subsection{UXO survey at detection test facility}

The objective of the first survey was to ascertain the performance of the two first movement-derived differences for UXO detection $\left(\Delta^{1} d\right.$, $\Delta^{2} d$ for both single and dual sensor cases, and additionally $\Delta^{3} d$ in the dual sensor case), as well as the relative cost of UXO recovery, when using the suggested potential target detection scheme. The survey was conducted at a UXO detection test facility in Denmark, where 12 total disarmed UXO, comprised of seven different types, were buried at depths between 0.5 and $1.3 \mathrm{~m}$ in an area spanning approximately $150 \mathrm{x}$ $250 \mathrm{~m}$. All of the UXO have been disarmed and salvaged from Danish waters, and all of them originate from the Second World War. The buried UXO are described in Table 3, and shown in Fig. 2. The test facility comprises a hilly field build of glacial till, including various pieces of metal scrap and a predominantly east-west buried pipeline. The survey was draped over the terrain, that is, flown at constant altitude relative to local ground level elevation, resulting in a ground level clearance of $\sim 1 \mathrm{~m}$ for the lower sensor throughout. An overview of the collected data from the test facility is provided in Fig. 3.

The first few movement-derived differences, with UXO locations overlain, are shown in Figs 4-6, for the dual, lower and upper sensors, respectively. Although our objective is limited to the first two orders of movement-derived differences, we also provide the third order for completeness, which highlights the existence of non-linear noise (particularly visible in Fig. 6). A close-up view of $\Delta^{1} d$ through $\Delta^{3} d$ near UXO 1, 2 and 3 are shown in Fig. 7, as these are difficult to distinguish the previous figures (the colour map scale remain unaltered for each 
Table 3. Overview of buried UXO, their burial depths and recovered equivalent dipole moment amplitudes at the detection test facility. Depths are measured relative to the centre of the longest axis, with an expected measurement error of less than $5 \mathrm{~cm}$. The dipole moment amplitudes enable comparison between extent and amplitudes of the dipolar part of the magnetic signatures, but do not generally, on their own, provide information regarding magnetic signatures shapes, nor any general information regarding size or shape of the UXO. The dipole moment estimates were obtained by collecting inversion results from the three most robust approaches. Error estimates are absolute errors, that is, all aforementioned results lie within the stated error limits.

\begin{tabular}{llcc}
\hline \multicolumn{1}{c}{ Number } & \multicolumn{1}{c}{ Type } & Depth $(\mathrm{m})$ & Dipole moment $\left(\mathrm{A} \mathrm{m}^{-1}\right)$ \\
\hline 1 & 105 mm shell & 0.50 & $0.29 \pm 0.06$ \\
2 & $75 \mathrm{~mm}$ canister, $20 \mathrm{~cm}$ long & 0.50 & $0.18 \pm 0.06$ \\
3 & $75 \mathrm{~mm}$ canister, $20 \mathrm{~cm}$ long & 0.50 & $0.05 \pm 0.01$ \\
4 & Naval mine without anchor, $130 \mathrm{~cm}$ diameter & 1.30 & $10.60 \pm 1.57$ \\
5 & 155 mm shell (very rusty) & 0.60 & $8.59 \pm 0.30$ \\
6 & AN-M30 or similar & 0.60 & $4.26 \pm 0.46$ \\
7 & MK40 or similar & 0.95 & $6.49 \pm 1.66$ \\
8 & 155 mm shell & 0.80 & $1.11 \pm 0.12$ \\
9,10 & Dual 105 mm shells & 0.70 & $0.98 \pm 0.10$ \\
11 & MK40 or similar & 0.90 & $4.69 \pm 0.75$ \\
12 & 155 mm shell & 0.75 & $2.07 \pm 0.35$ \\
\hline
\end{tabular}
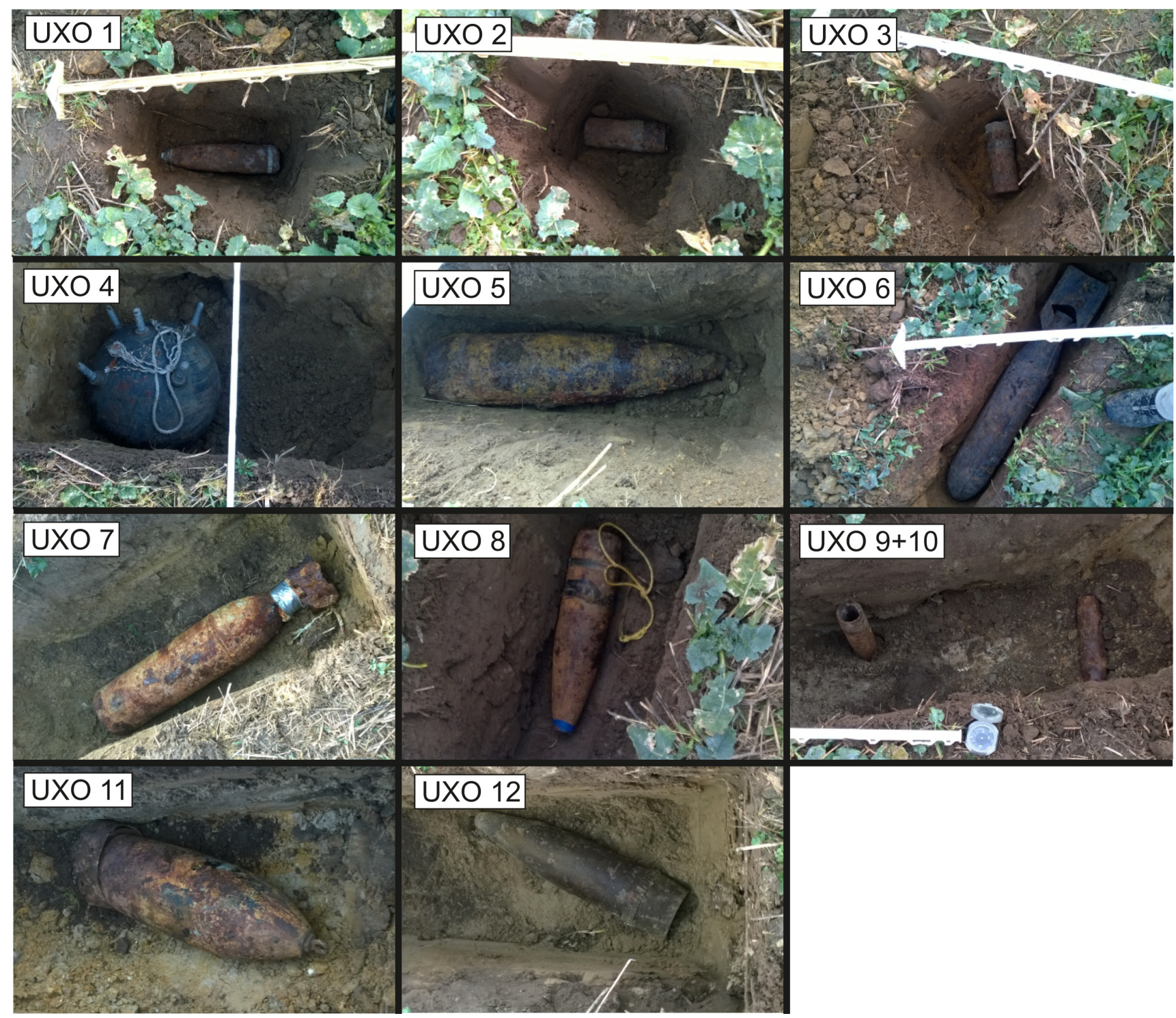

Figure 2. UXO buried at the test facility. Numbers in panel corners correspond to the UXO numbers listed in Table 3. 
Raw data (Upper sensor)



Raw data (Lower sensor)

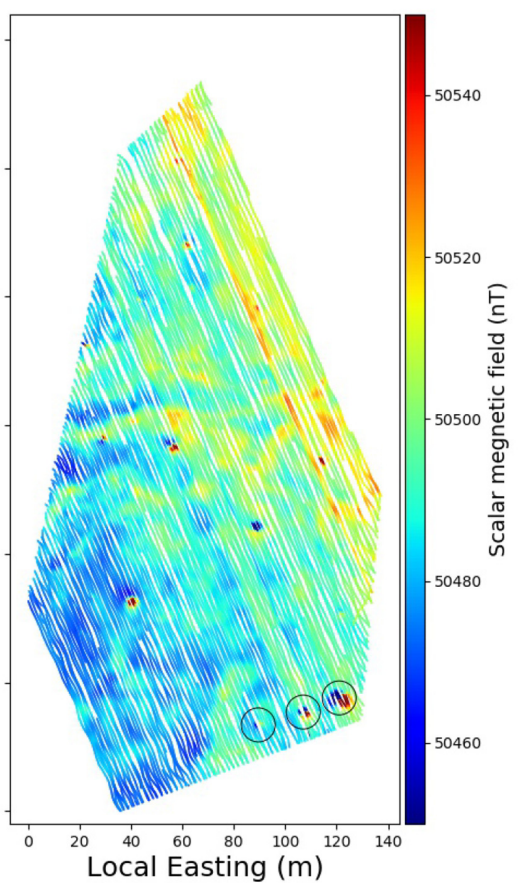

Deviation from vertical ray

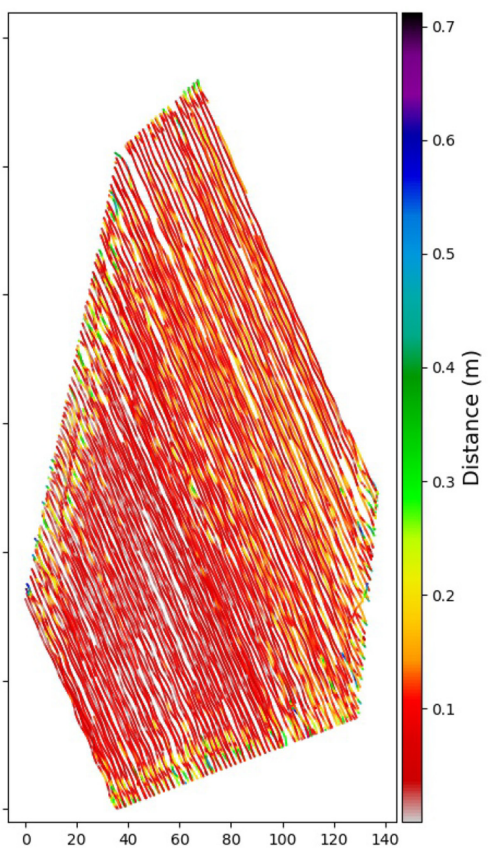

Figure 3. Raw data from the UXO test facility, as collected by the two sensors. The three marks each correspond to a deliberately placed piece of metal scrap. Left-hand pane: upper magnetometer signal. Centre pane: lower magnetometer signal. Right-hand pane: horizontal offset of the lower sensor with respect to the upper sensor, defined as the distance between their projections on a plane that is perpendicular to the gravitational acceleration vector. The horizontal offset is calculated from the attitude data, resulting in a maximum uncertainty of $\sim 2 \mathrm{~cm}$.

$\Delta^{1}$ (Dual sensor)

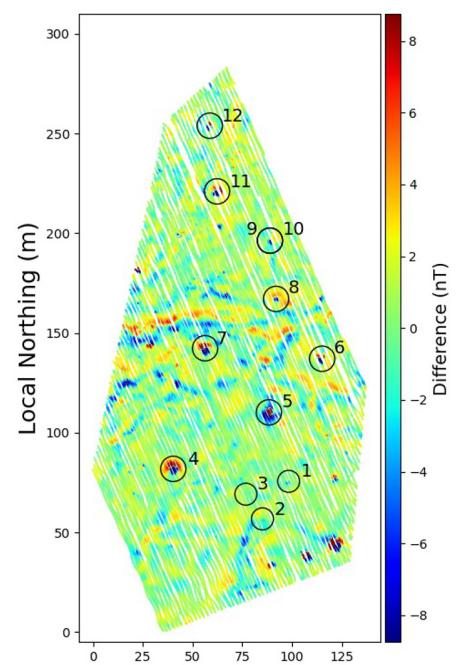

$\Delta^{2}$ (Dual sensor)

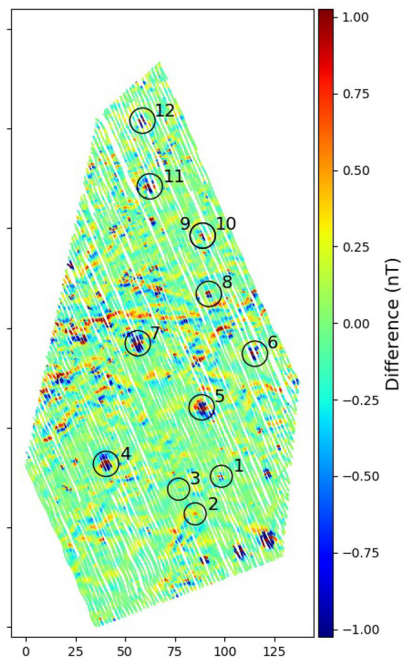

$\Delta^{3}$ (Dual sensor)

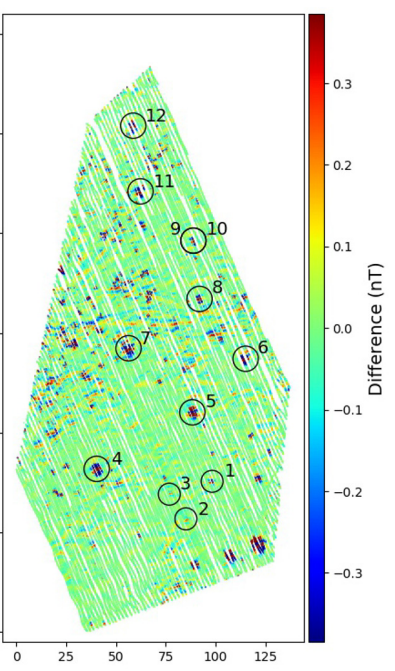

$\Delta^{4}$ (Dual sensor)

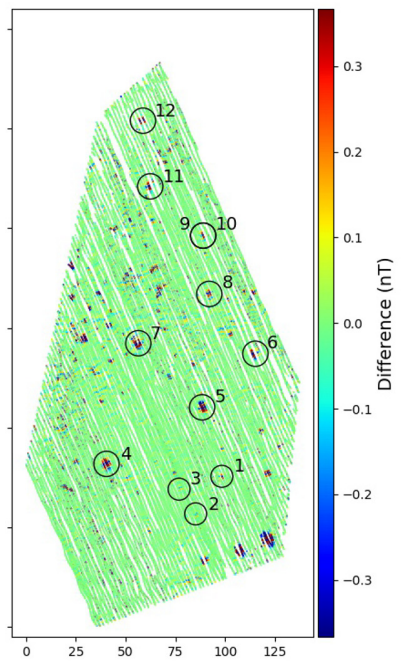

Local Easting $(\mathrm{m})$

Figure 4. Comparison of dual sensor differences at the UXO test facility. Colour scales have been set to \pm 10 times the MAD of the data. Differences with an uneven number of movement-based components have been adjusted for travel direction, that is, data have been multiplied by -1 on every other line. Left-hand pane: the first $(k=1)$ difference across the two sensors. Centre left pane: the second $(k=2)$ difference, which is obtained by computing chained differences of $\Delta^{1} \mathbf{d}$ in the flight direction. Centre right pane: the third $(k=3)$ difference, which is obtained by computing the second chained difference of $\Delta^{1} \mathbf{d}$ in the flight direction. Right-hand pane: the fourth difference (i.e. the third movement-derived difference in the dual sensor case).

difference). The accentuation of shorter wavelengths with increasing $k$ is evident: $k=1$ accentuates a substantial part of the short wavelengths visible in the raw data and obscures longer wavelengths, while higher order differences amplify this property until the noise limit is reached, which occurs at the third movement-derived difference (by far most pronounced on $\Delta^{3} d$ of the lower sensor data, but also slightly discernible on some lines of $\Delta^{3} d$ of the lower sensor, and $\Delta^{4} d$ when utilizing dual sensors). UXO with larger magnetic signatures were easily discernible, and were recovered irrespective of sensor configuration for the first few differences. The first order differences $k=1$ were unable to recover 


\section{$\Delta^{1}$ (Lower sensor)}

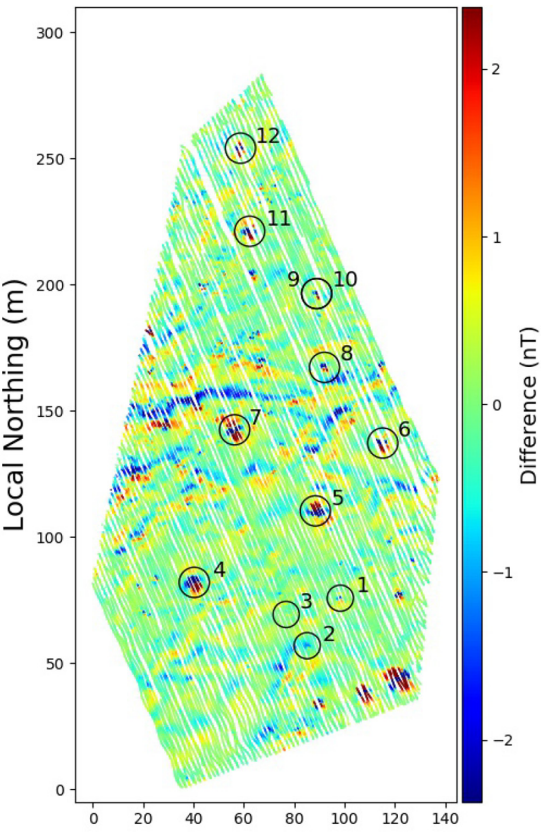

$\Delta^{2}$ (Lower sensor)

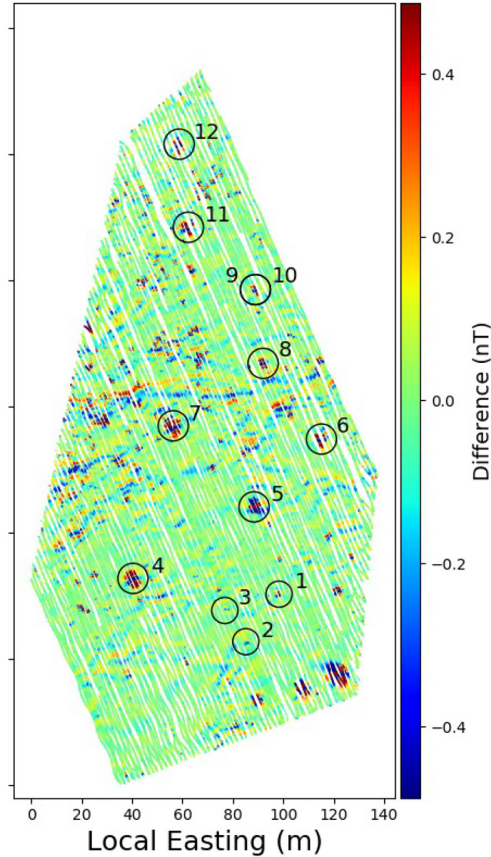

$\Delta^{3}$ (Lower sensor)

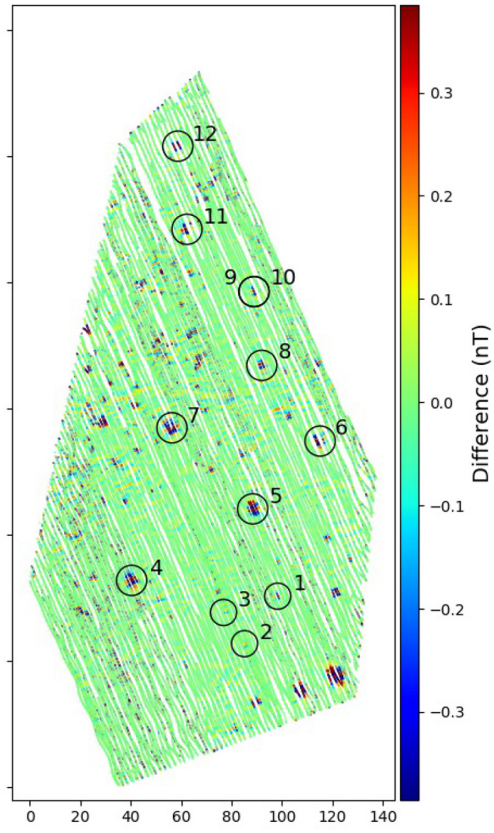

Figure 5. Comparison of lower-sensor differences at the UXO test facility. Colour scales have been set to \pm 10 times the MAD of the data. Differences with an uneven number of movement based components have been adjusted for travel direction, that is, data have been multiplied by -1 on every other line. Left-hand pane: the first $(k=1)$ difference across adjacent measurements collected by the lower sensor. Centre pane: the second $(k=2)$ difference, which is obtained by computing chained differences of $\Delta^{1} \mathbf{d}$ in the flight direction. Right-hand pane: the third $(k=3)$ difference, which is obtained by computing chained differences of $\Delta^{2} \mathbf{d}$ in the flight direction.

\section{$\Delta^{1}$ (Upper sensor)}

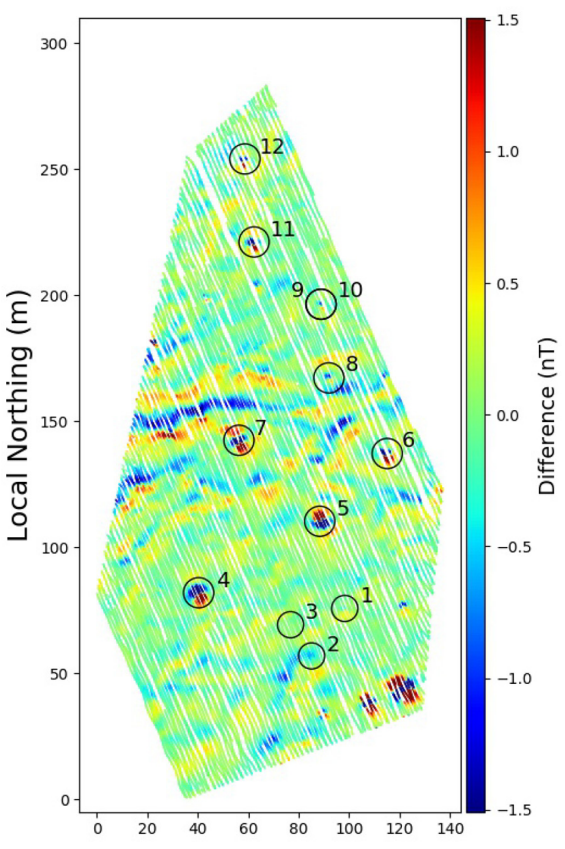

$\Delta^{2}$ (Upper sensor)

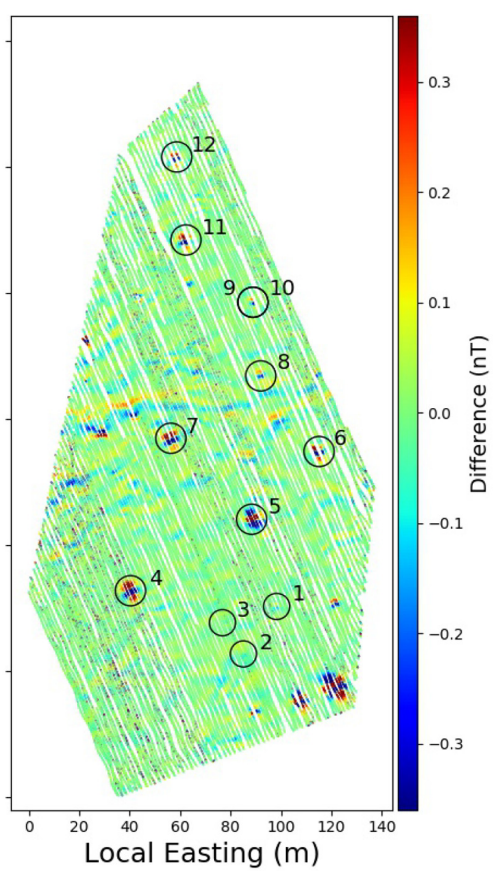

$\Delta^{3}$ (Upper sensor)

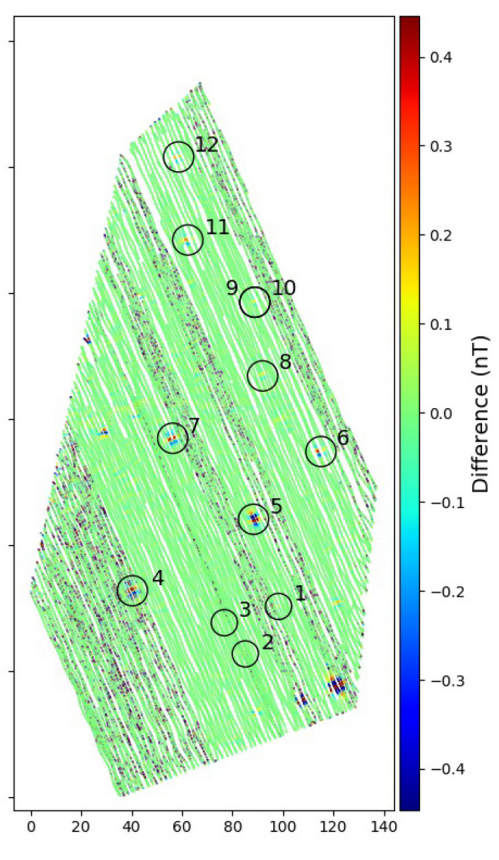

Figure 6. Comparison of upper-sensor differences at the UXO test facility. Colour scales have been set to \pm 10 times the MAD of the data. Differences with an uneven number of movement based components have been adjusted for travel direction, that is, data have been multiplied by -1 on every other line. Left-hand pane: the first $(k=1)$ difference across adjacent measurements collected by the lower sensor. Centre pane: the second $(k=2)$ difference, which is obtained by computing chained differences of $\Delta^{1} \mathbf{d}$ in the flight direction. Right-hand pane: the third $(k=3)$ difference, which is obtained by computing chained differences of $\Delta^{2} \mathbf{d}$ in the flight direction. 




Figure 7. Comparison of dual sensor differences for the three smallest UXO at the test facility. Columns correspond to difference orders and rows correspond to sensor choice. Note that the first double sensor difference is computed across sensors only. All colour scales are set to \pm 10 times the MAD of the entire survey for the corresponding difference. Differences with an uneven number of movement based components have been adjusted for travel direction, that is, data have been multiplied by -1 on every other line.

the smallest UXO (nos 1-3 in Table 3) with the proposed inversion approach, but these could be recovered using higher order differences. Of the three UXO, UXO 1 exhibited the highest dynamic range across all tests, while UXO 3 exhibited the smallest, and was always the most challenging UXO to recover. An overview of threshold peak prominence values and their relative costs (amount of non-UXO targets recovered at the same threshold), are shown in Table 4, while a graphical overview of the relative detection costs are provided in Fig. 11. Further details on the exact detection limits of various UXO is provided in the Supporting Information (SI). The standard deviation is potentially interesting for UXO detection, as it can serve as the peak prominence threshold in the dual-peak method. We also include the MAD (median absolute deviation) mainly to provide insight into the combined background variation and noise envelope levels in each data product. Differences where the noise limit is clearly visible on colour scales of $\pm 10 \times \mathrm{MAD}$, that is, movement-derived differences of order three and above, are not considered. It should be noted that the cost of recovery of the smallest UXO at the test facility may not reflect the general detection scenario, as this will depend on parameters specific to the survey location, including the complexity and amount of non-UXO targets, as well as the survey altitude. In general for UXO surveys, non-UXO objects of similar magnetic amplitude are expected, and thus additional discrimination procedures may be necessary if such small UXO are to be considered.

To evaluate performance inversion robustness, that is, if the UXO are recoverable with the proposed approach, and how good an initial position guess is required for convergence to the UXO position, we invert each UXO from multiple different initial positions. Variations in magnetic susceptibility and permanent magnetization hamper meaningful prediction of the equivalent dipole moment, especially when the type of UXO is not known a priori. We therefore only test the inversion process using the initially proposed unit dipole moment directed along the local core field (which proves sufficient for all UXO at the test facility).

We select four different horizontal offsets (radii) from the UXO; 0.25, 0.5, 1 and $2 \mathrm{~m}$. Eight points are placed per radii, $45^{\circ}$ part. A visualization of the resultant horizontal point distribution is shown in Fig. 8. We also vary the initial depth estimate, as well as the data inclusion radius (i.e. the radius around the initial guess, in which data are retained for the inversion). Aggregate robustness test results are provided in Fig. 9, while recovered depth estimates are shown in Fig. 10. A visualization of recovery percentages for individual UXO is provided in Fig. 11. Inversion on higher order differences, namely $k=2$ in the lower sensor case, and both $k=2$ and 3 in the dual sensor case, proved sufficient to recover all UXO, and also retained the highest degrees of inversion robustness across all methods. For larger UXO, 
Table 4. Standard deviation and MAD of the difference data products. Also shown are the total targets recovered (combined amount of UXO and non-UXO objects with anomaly prominences above the standard deviation or MAD, respectively) and resultant percentage of UXO in the total targets recovered, that is, the percentage cost of detection. Three of the detected non-UXO anomalies are deliberately placed scrap metal objects, and thus the listed numbers contain a small bias. Since UXO 9 and 10 are always recovered as a single anomaly, we count them together as a single target for all purposes.

\begin{tabular}{lcccccc}
\hline Entity & \multicolumn{3}{c}{ Std. dev. } & \multicolumn{3}{c}{ MAD } \\
& Std. dev. (nT) & Targets & $\begin{array}{c}\text { UXO (per } \\
\text { cent) }\end{array}$ & MAD (nT) & Targets & $\begin{array}{c}\text { UXO (per } \\
\text { cent) }\end{array}$ \\
\hline$\Delta^{1} D$ & 9.98 & 30 & 20.0 & 0.87 & 750 & 1.5 \\
$\Delta^{1} L$ & 3.82 & 46 & 17.4 & 0.24 & 940 & 1.2 \\
$\Delta^{1} U$ & 0.89 & 223 & 4.5 & 0.15 & 877 & 1.3 \\
$\Delta^{2} D$ & 3.11 & 28 & 28.6 & 0.10 & 1000 & 1.1 \\
$\Delta^{2} L$ & 1.77 & 36 & 22.2 & 0.05 & 835 & 1.3 \\
$\Delta^{2} U$ & 0.27 & 575 & 1.9 & 0.04 & 762 & 1.4 \\
$\Delta^{3} D$ & 1.55 & 35 & 22.9 & 0.04 & 691 & 1.6 \\
\hline
\end{tabular}

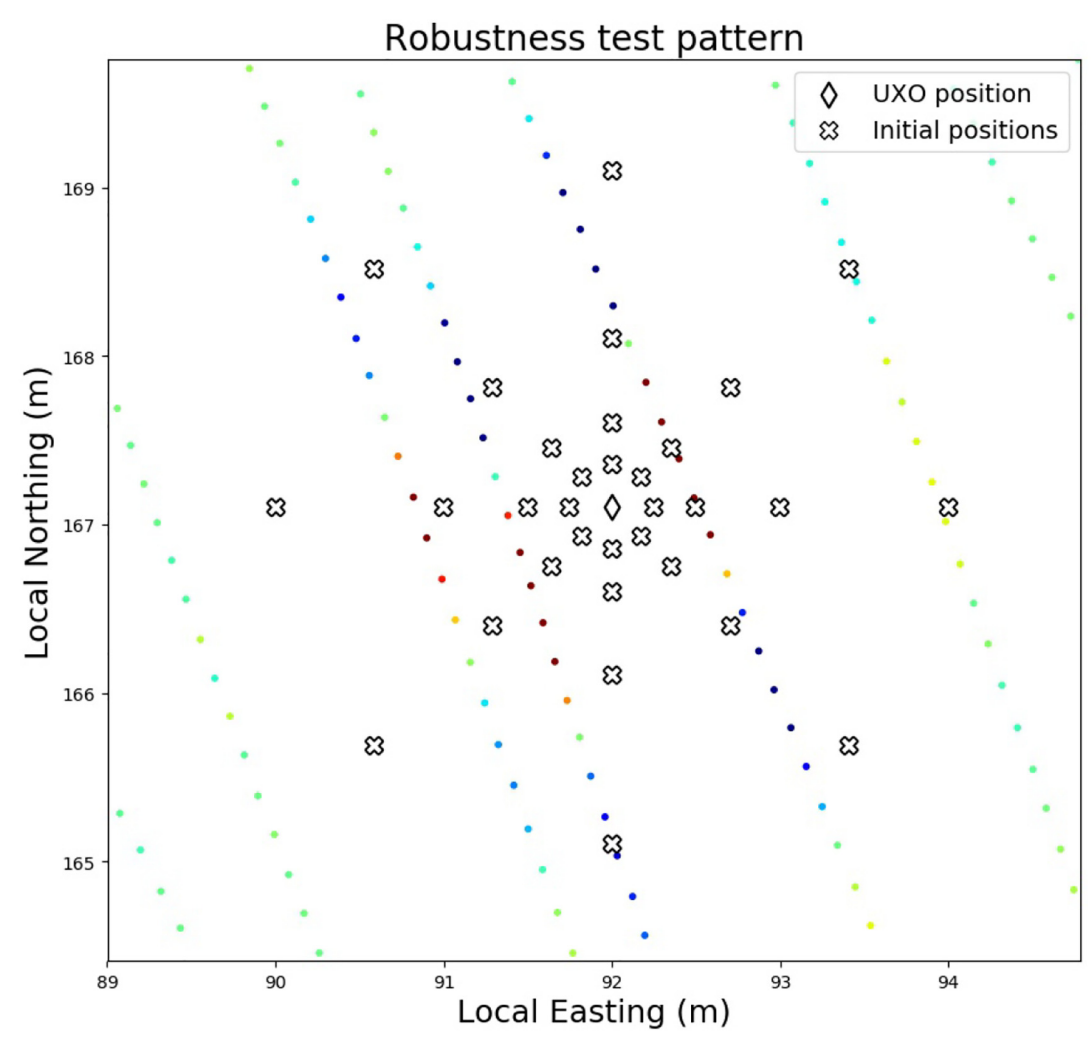

Figure 8. Example of robustness test strategy for a given vertical coordinate and UXO signature. An inversion was attempted for each of the 32 points, which are placed $45^{\circ}$ apart horizontally in four distinct radii $(25,50,100$ and $200 \mathrm{~cm})$, from the actual horizontal position of the UXO.

$k=1$ in the dual sensor case, and $k=2$ in the upper sensor case, proved sufficient for detection if a large enough data radius was retained. Regardless of the employed method, UXO 9 and 10 were always recovered as a single anomaly. Numerous non-UXO targets with seemingly dipolar signatures were present at the test facility, and a multitude of these were recovered using the proposed two-peak method, as shown in Table 4. We make no attempts at classification nor inversion robustness testing on any non-UXO anomalies; this is beyond the scope of the study.

\subsection{Coastal UXO survey in relation to the Hornsea II Offshore wind farm}

A commercial magnetic survey was conducted with the measurement system in the Spring of 2019, in relation to cable routing for the Hornsea II Offshore wind farm, off the eastern coast of Great Britain. The surveyed area consists of two parallel rectangular areas which were deemed inaccessible with conventional surveying methods, and therefore needed to be covered with the UAV-borne system. The inner, approximately $140 \mathrm{~m} \times 500 \mathrm{~m}$ area consists of interwoven intertidal and shallow-water/surf zone, while the outer, approximately $100 \mathrm{~m} \times 500 \mathrm{~m}$ area is 


\section{Percentage of UXO in targets recovered at UXO detection thresholds}
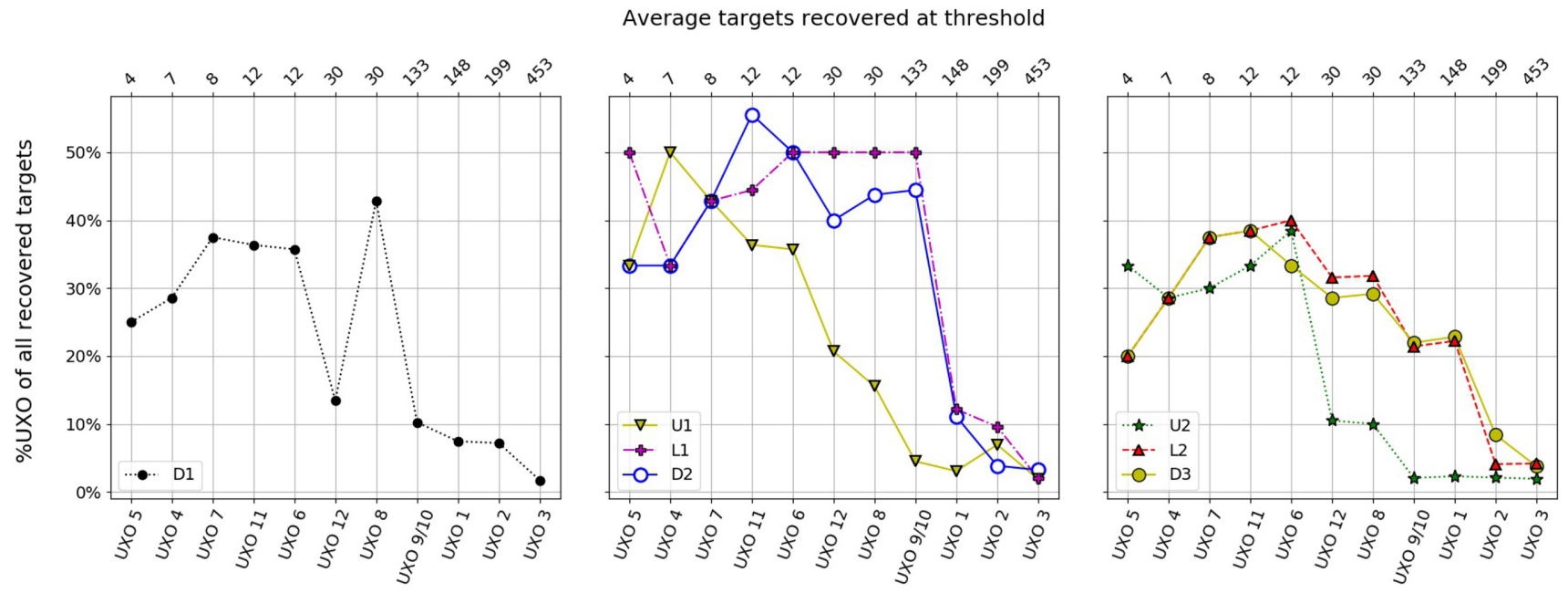

UXO recovered (cumulative)

Figure 9. Convergence results from the robustness test performed on test facility data. Bar values were obtained by stacking results from tests using 3,5 and $7 \mathrm{~m}$ data inclusion radii. Letter codes refer to upper sensor (U), lower sensor (L) and double sensors (D), respectively. Numbers following each letter correspond to the differencing order $(\mathrm{k})$. Bar colours represent horizontal offsets between the initial horizontal position guess and the UXO position, while individual subplots represent vertical offsets with respect to the actual UXO burial depth. Note that U1 and L1 have identical results. Further details on the convergence results are provided in the SI.

Aggregate convergence results for varying initial depths and horizontal offsets

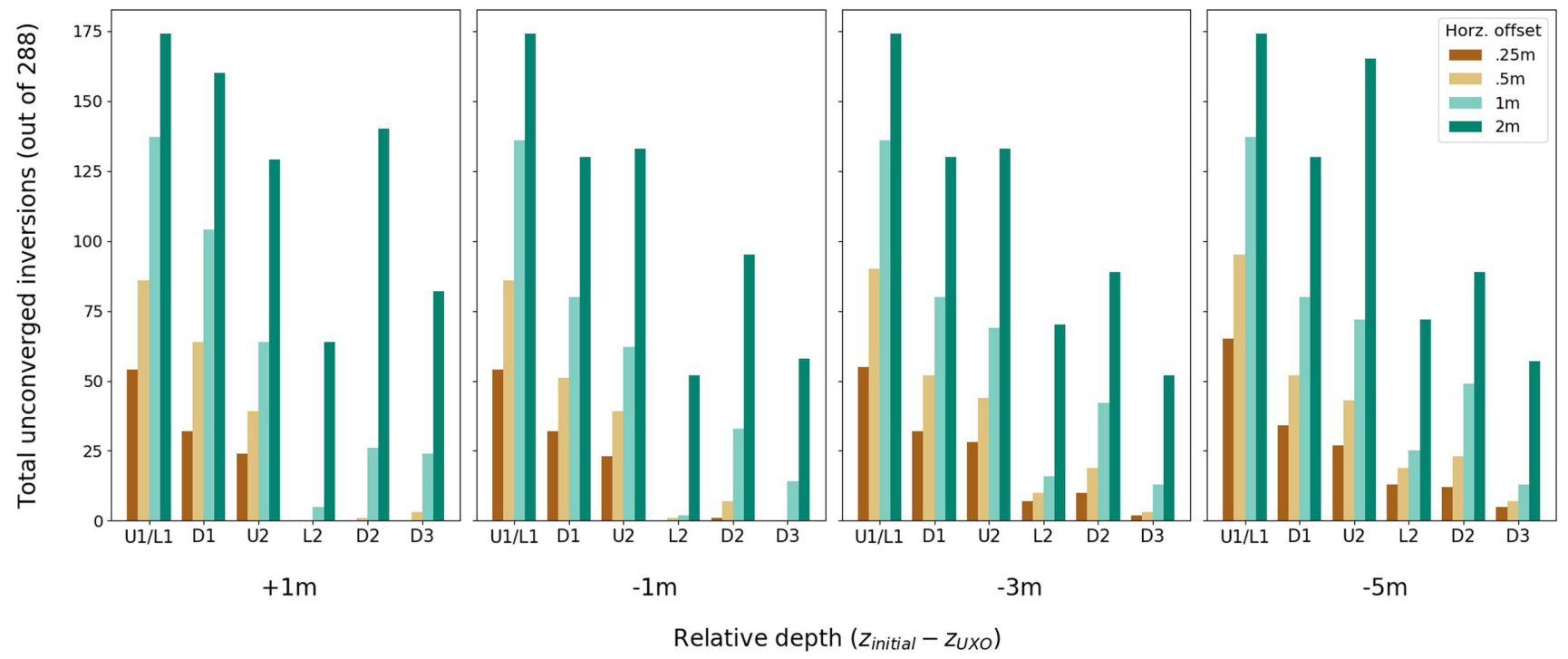

Figure 10. Depth estimate errors achieved with different methods on test facility data, for each individual UXO. The upper row of graphs contain methods unable to converge to a position near every single UXO, while the lower row of graphs contain the methods which successfully achieved convergence at each UXO. Note the significant change in robustness across the methods: each graph in the lower row exhibits a highly similar pattern, with only minute exceptions in L2 and D2 when using a $3 \mathrm{~m}$ data inclusion radius. In the upper row, D1 and U2 are robust between 5 and $7 \mathrm{~m}$ data radii, while most of the remaining depth errors vary. Note the increase of very deep depth estimates with increasing data inclusion radius in the upper row, which also tends to increase the maximum depth errors ( $2.4 \mathrm{~m}$ in the upper left pane, $5.9 \mathrm{~m}$ in the centre pane and $9.1 \mathrm{~m}$ in the right-hand pane). These outliers stem from the inability to fit the UXO signatures, due to them being drowned out by other remaining signals in the data, which are fitted instead. The highest depth robustness across all data inclusion radii was obtained with $\Delta^{3} D$.

a shallow-water/surf zone generated by a submerged sandbank. Due to limited windows of operation, data collection was spread out over several days. Wind conditions varied across operational windows, as well as within them. The onsite tidal variation was in excess of $6 \mathrm{~m}$, with surveying limited to water levels $<2 \mathrm{~m}$ above the low tide. As the seabed depth was not explicitly known, we set the initial depth estimate at $1.5 \mathrm{~m}$ below the lowest observed sea surface height, based on a heuristic approximation from the beach slope at the UAV take-off location. Due to the expected distance between sensor and source, the large spatial extents of some of the observed anomalies, and the relatively 
Data inclusion radius $=3 \mathrm{~m}$
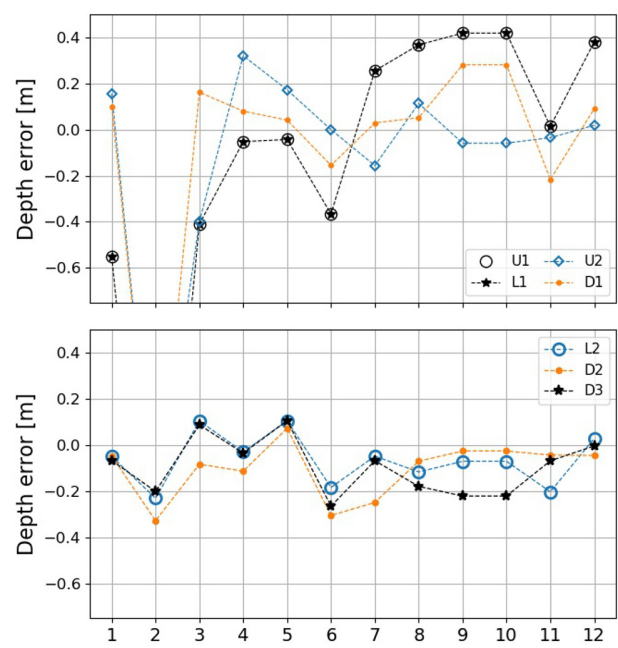

Data inclusion radius $=5 \mathrm{~m}$
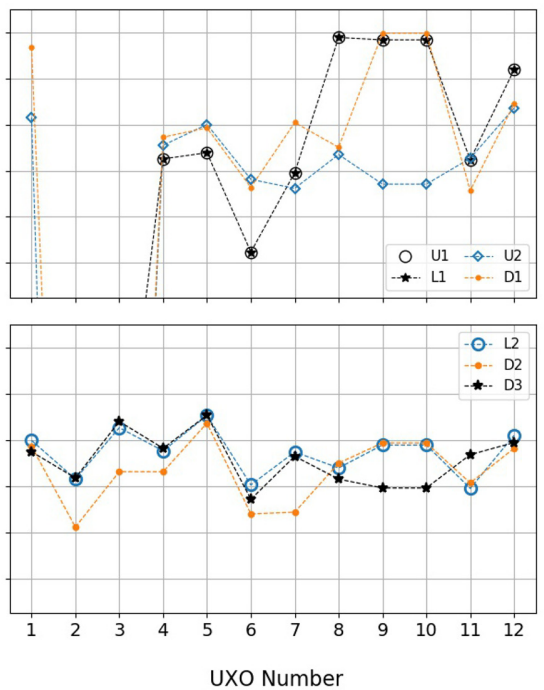

Data inclusion radius $=7 \mathrm{~m}$
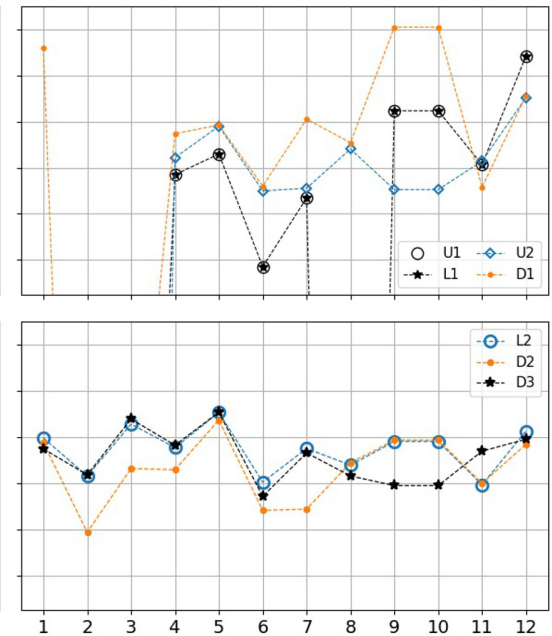

Figure 11. Percentage of UXO in the total amount of potential targets for the detection limit of each UXO. Letter codes refer to upper sensor (U), lower sensor (L) and double sensors (D), respectively. Numbers following each letter correspond to the total differencing order (k). A value of 50 per cent for a given UXO informs us that if we dig at every target position that was recovered at the recovery threshold of that UXO, we will find UXO in half of the holes dug. Thus, higher values correspond to lower detection costs (i.e. fewer holes need to be dug per detected UXO), and vice versa. Note that apart from the buried UXO, three of the detected anomalies are deliberately placed scrap metal objects, and thus there is a small negative bias in the listed percentages. Since UXO 9 and 10 are always recovered as a single anomaly, we count them together as a single target for all purposes. Data products (differences) have been grouped based on the amount of movement-derived orders they contain. Left-hand pane: data products containing no (zeroth order) movement-derived differences. Centre pane: data products with first order movement-derived differences. Right-hand pane: data products with two orders of movement-derived differences.

large spatial distance between neighbouring targets, we select a data inclusion radius of $10 \mathrm{~m}$. The initial horizontal positions and dipole moment direction guesses were obtained as previously described, with one alteration: due to the presence of high-frequency noise in the data (particularly visible at higher order differences, as in the test facility data), we apply a moving mean filter in the temporal dimension over five samples, and use the resultant smoothed map to distinguish potential targets. The inversion was still performed on the original (pre-processed) data.

Inversion results with the initial horizontal positions were rejected in two cases: Ii) the depth estimate was infeasible, that is, placed above the water surface and (ii) the obtained horizontal position placed the target outside the radius of data considered, or outside the data region. An overview of the dual sensor magnetic difference, the horizontal deviation of sensors from vertical alignment, and the sensor system pitch angle, are shown in Fig. 12. The first single and second dual sensor differences, including detection results when utilizing their respective standard deviations, are shown in Fig. 13.

Note the large deviations in horizontal position between the two sensors throughout the survey in Fig. 12. This is mainly due to local wind conditions. Strong winds and gusts directed along the positive $Y$-axis enforced variations in sensor position across neighbouring lines, which is clearly seen as a striping pattern in the pitch data.

The data and detection results obtained from the lower and dual sensors in Fig. 13 are generally similar. The lower sensor detects one target which the dual sensor does not (upper left corner), while the dual sensor approach detects six that the lower sensor does not (mainly in the lower half of the survey). There are a few very weak, seemingly dipole-shaped anomalies, which remain undetected by either method, as they encompass a variation of less than one standard deviation of the data (and thus are never considered potential targets in the first place).

A total of eight targets were investigated in-depth following the UXO survey, all of which corresponded to a non-UXO metal object. The location of each of these eight targets were accurately predicted by all differences computed from the HOW02 data.

\section{DISCUSSION}

The scalar magnetic difference method provides a highly versatile, computationally simple framework for direct source inversion, for any amount and configuration of sensors, provided that the sensor positions are known. When used with a single sensor, time-varying external signals must be considered, while for two or more synchronized sensors, recovered differences will be largely invariant to external noise, for example, diurnal variations. While the external geomagnetic field variations could be handled by deploying a base station magnetometer in the vicinity of the survey area, the method is fragile to local field variations, for example, in urban settings where the distance between base 


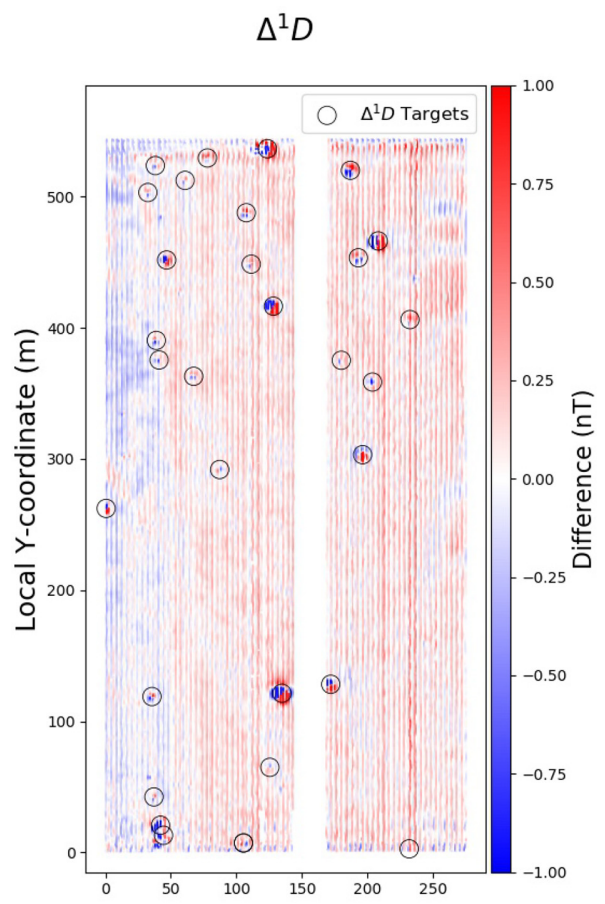

Deviation from vertical axis

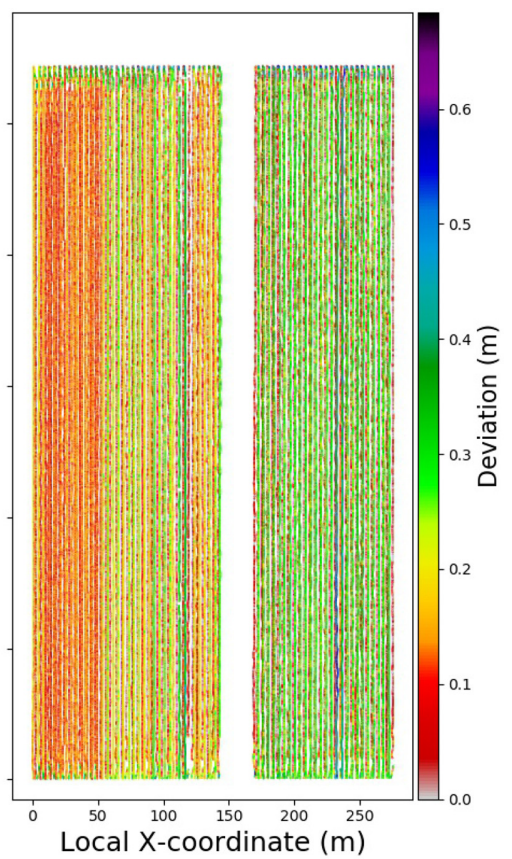

Pitch angle

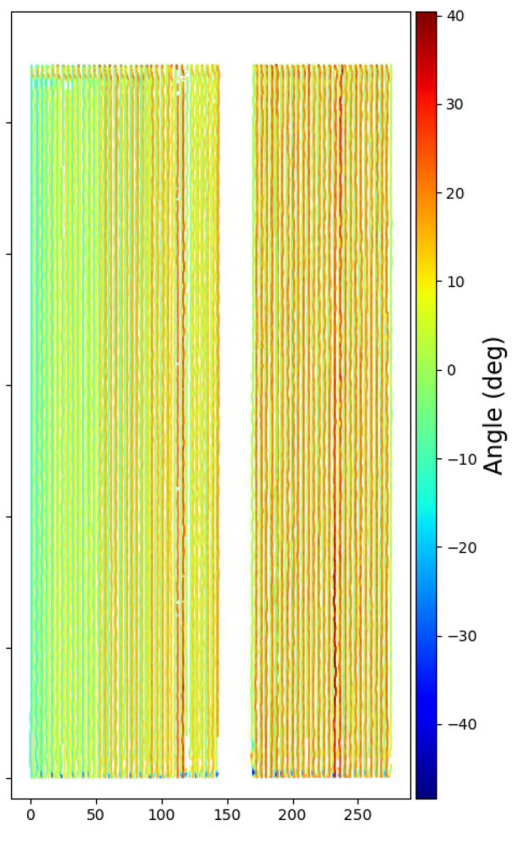

Figure 12. Dual sensor difference collected in the coastal survey. Left-hand pane: the measured scalar magnetic difference, with detected targets overlain. The smallest dipole estimate among targets is $0.20 \mathrm{~A} \mathrm{~m}^{-1}$. Centre pane: horizontal offset of the lower sensor with respect to the upper sensor, defined as the distance between their projections on a plane that is perpendicular to the gravitational acceleration vector. The horizontal offset is calculated from the attitude data, resulting in a maximum uncertainty of $\sim 2 \mathrm{~cm}$. Right-hand pane: angle of pitch angle of the sensor towing system. The root mean square pitch angle uncertainty is $1^{\circ}$. Note that the front of the system is always facing in the direction of the positive $y$-coordinate.

$\Delta^{1} \mathrm{~L}$

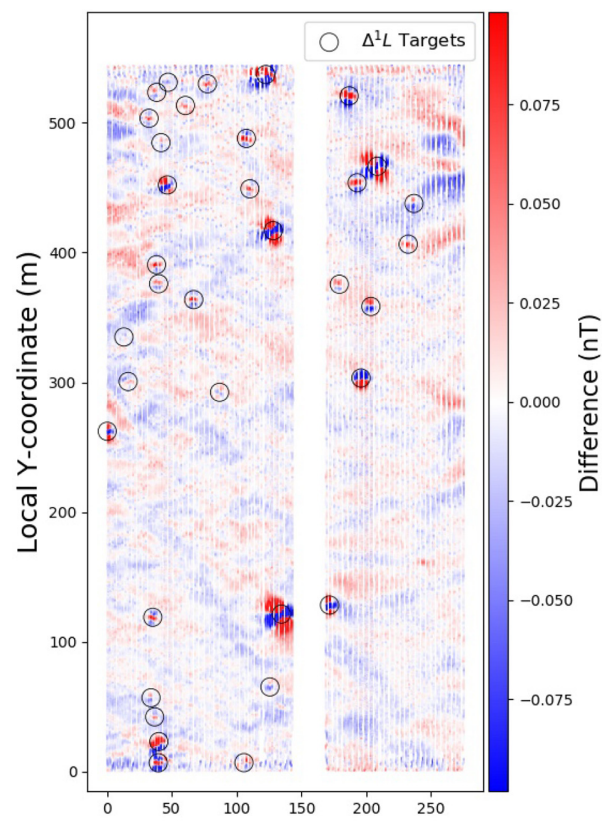

$\Delta^{2} \mathrm{D}$

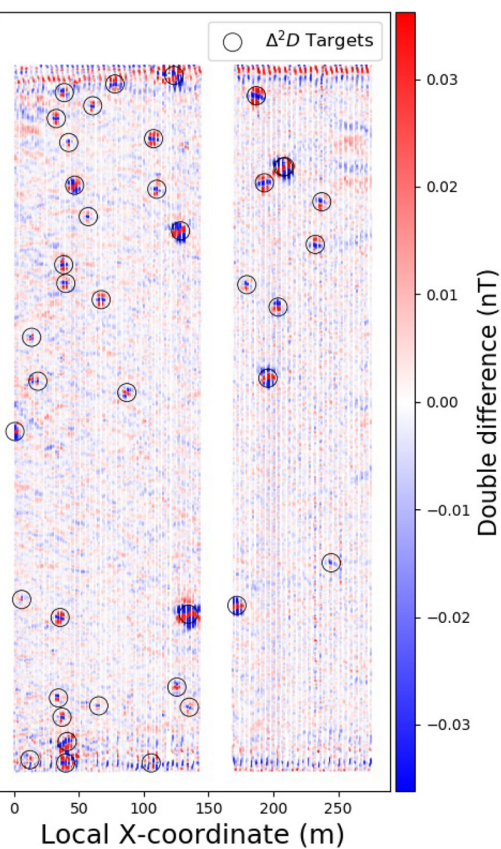

Target comparison

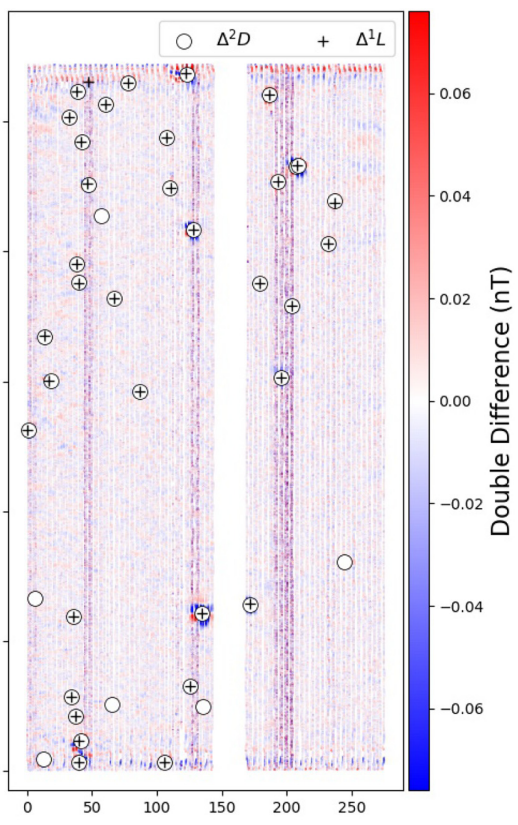

Figure 13. Targets detected in the coastal survey using the first and second difference of the lower and dual sensors, respectively, that is, $\Delta^{1} L$ and $\Delta^{2} D$, each of which contain one movement-derived difference. Targets were detected using the described method with a peak prominence requirement of one data standard deviation. Left-hand pane: lower sensor difference with detected targets overlain. The smallest dipole estimate among detected targets is $44 \times 10^{-2}$ $\mathrm{A} \mathrm{m}^{-1}$. Centre pane: dual sensor double difference (one across the two sensors, and one along the survey direction), with targets overlain. The smallest dipole estimate among detected targets is $25 \times 10^{-2} \mathrm{~A} \mathrm{~m}^{-1}$. Right-hand pane: unfiltered dual sensor double difference with targets from both methods overlain, for comparison. The high-frequency noise visible on the unfiltered dual sensor difference is also visible in the unfiltered lower sensor difference. 
station and roving sensor cannot be assumed negligible. In such scenarios, the difference between two sensors is arguably far more robust to external noise, as the underlying assumptions behind the method will only break down in far more strenuous circumstances, for example, the bias sources will need to be in the immediate vicinity of the system (or stem from the system itself). For magnetometers with high sampling rates, however, it may be prudent to compute differences between measurements over a select distance, as adjacent measurements may not be able to recover target signature amplitudes larger than the noise level. The main strengths of the method are broad and direct applicability, and the ability for rapid processing. As such, the method may be applied to handle common commercial challenges such as rapid turnaround times for data deliverables (usually $24 \mathrm{hr}$ ), where conventional filtering and and manual data adjustments may be too time consuming.

While the presented data are arguably of high quality, a high-frequency bias is visible on higher order differences of the data. The bias signal amplitude differs across the upper and lower sensors, being higher in the former, which suggests a bias source above the two sensors. Interestingly, the bias signal only occurs in specific groupings of survey lines, suggesting that the bias signal is enabled and disabled during landings. We suspect that this may be due to either a loose connection in the sensor system (one was discovered subsequently), sporadic disturbance from the navigation payload, or from the UAV itself, but the specific source remains a mystery. Regardless, results from the test facility suggests that scalar magnetic differences can be reliably employed for magnetic target detection, and we successfully retrieved and positions of all buried targets with higher order differences.

In the test facility data, higher order differences are more robust to changes in initial conditions, more reliably recovering the same optimum in the inversion. Furthermore, depth estimates of the lower and double sensor differences are very robust, even for small data inclusion radii, enabling fast inversion. Choice of a single best method is controversial, particularly in the presence of a high-frequency bias emanating from the system itself, and due to the low ground clearance. In general, we see an increase of both total amount of targets detected, and inversion robustness with increasing $k$. While the optimal order of $k$ for a given survey may vary, the long wavelength dampening effect of higher differences seem to result in heightened inversion robustness, that is, it may be worth considering to use the highest order difference possible without needing to filter, transform, downsample, or otherwise tamper with the data.

The twice movement-derived differences of the lower and dual sensors, that is, $\Delta^{2} L$ and $\Delta^{3} D$ both provide excellent results at the test area, but for the former in particular this may partially stem from the low ground clearance and smaller bias signal. In the coastal survey data, the first movement-derived differences, that is, $\Delta^{1} L$ and $\Delta^{2} D$, detect a different amount of anomalies, but this should be considered a property of the potential target detection routine, rather than a direct property of the differences. It is clear, however, that the addition of an extra sensor in this case enables further dampening of the longer wavelength signals, as particularly visible in the northeastern part of the coastal survey.

We elect to stop at the second and first movement-derived differences in the test facility and coastal data, respectively, as we are nearing the noise floor of the data. Higher order differences are possible (especially in the dual sensor case), but require some degree of filtering, which in turn biases the data relative to the model predictions. If data filtering (or alternative transformation) should be necessary, one approach may be to filter both the synthetic (predicted) data analogously to the measured data, which is beyond the scope of this study.

We have considered target detection, that is, the question of whether a magnetically significant target is present in the data. It is also worth noting that we have made no attempts at UXO discrimination, apart from using the depth estimate to discard select anomalies placed at infeasible positions in space, or when discarding results outside the data region. In relation to the depth estimate, we find a high degree of agreement between both the lower and dual sensor differences in either survey, and that these estimates correspond well to the measured depth estimates. Although the standard deviation has been used as a threshold parameter throughout this study, it cannot be directly taken as a general rule of thumb. The reason that the standard deviation works as threshold parameter in this study is mainly due to its property of being biased by outliers, generally resulting in the threshold being somewhere between the strongest peaks and any well-defined baseline in the considered data or data product (if such a baseline exists). It follows that the approach may only work if the target signatures (in this case UXO) are scarce and significantly larger than the noise level or geological background, whichever is dominant. As such, this approach remains somewhat arbitrary, as it is largely dependent on the relationship between targets and baseline, and may easily breakdown in more complex scenarios. In practice, the choice of threshold should generally depend on multiple factors such as the survey scenario, survey objective and data quality, for example, by considering a priori information regarding survey site and expected targets, as is commonplace in the industry.

The use of the standard deviation as peak prominence limit is based in the reasoning that anomaly signatures will carry significant power compared to the background, that is, the distribution of the data will be long-tailed. In such cases, the standard deviation will be a biased estimate of the spread parameter, which we count on for threshold selection, that is, the standard deviation is best suited for detecting the largest anomalies. However, for data with a clean magnetic background, as in the coastal data set, the standard deviation approach locates targets with far smaller equivalent dipole moments, that is, the level of when to consider a target significant has changed. It is here important to consider the application perspective, as smaller UXO may not necessarily be particularly interesting from, for example, a commercial point of view, and thus a completely different criteria may be relevant (in fact, it is commonplace in some commercial UXO surveys to have a pre-specified limit based on known meta-parameters). The recovered dipole moments do not vary significantly across the higher order dual and lower sensor differences (as demonstrated by the dipole moment spans provided in Table 3). The dipole moment spans from Table 3 also show that the smallest UXO dipole moment recovered at the test facility, is larger than the smallest recovered anomalies in the coastal survey data. While the two-peak method may have resulted in the discarding of anomalies with equivalent dipole moments similar or slightly higher than the lowest recovered moment amplitude, we regard it to be highly unlikely that any significantly larger dipole moments have been 
missed. However, due to the vector field nature of the anomalies, it is impossible to be certain that the target list obtained from the coastal data set is exhaustive, and we can make no explicit comparison to targets from the test facility.

In the data positioning, we made the assumption that the axis on which the magnetometers are aligned always intersects the UAV as well. While we have observed this to be a good assumption in general during stable flight, this assumption breaks down during abrupt movements, such as during turns or when gusts of wind are encountered. Although the most abrupt movements, encountered during turns, were removed during data trimming, the occasional strong sporadic gusts were still encountered during the surveys. In such areas, it is possible that the vertical positioning accuracy of the data will be diminished. Furthermore, the intersect point on the UAV was not observed to be constant, due to slight oscillations of the payload, and as such there is most likely an added uncertainty from this assumption which has not been taken into account in the stated total horizontal and total vertical absolute uncertainties, as well as in the relative uncertainty. However, the observed oscillations were slow and of small amplitude, and we estimate these to account for $\leq 10 \mathrm{~cm}$ (absolute) offset at any given time, while being largely negligible with respect to the position drift between two adjacent samples. Furthermore, the coarse estimates of the drift in measured position between samples, which were primarily based on three small subsets of the data, cannot be expected to be unbiased, since the selected subsets may have been biased by, for example, wind, as well as non-ideal antenna orientations during pauses in the flight, that is, the experimental data were not collected in a sufficiently controlled environment.

While the target inversion method with the point-dipole model is generally applicable, it suffers from two main flaws. First, it provides no means of distinguishing between actual UXO and other magnetic targets, nor does it provide any information on the size of the target, as neither target susceptibility nor volume can be resolved. The implementation of the method in this study has been based around the choice of a somewhat arbitrary parameter, the peak prominence threshold, which determines the amount of potential targets in the data based on the peak prominences of their magnetic signatures. While once again generally applicable, the approach cannot guarantee detection, as the resolution of two peaks may not be feasible, for example, due to targets with small magnetic signature, or enveloped in complex background signals, as seen in the test facility data. We stress, that neither this method, not any other viable UXO detection method known by the authors, is able to guarantee the recovery of all present UXO.

Sway may have some influence on the measured difference due to difference in distances between each individual magnetometer and the UAV, potentially resulting in a difference in sway-induced error across the sensors, as is visible when comparing the left- and right-hand panes of Fig. 13. The same is true for the distance between each individual magnetometer and the navigation payload. However, as swaying of the magnetometers also introduces a change in the relative altitude, this is difficult to quantify, against anything but a very well-known background. The results of Walter et al. (2019a) report a deviation of up to $0.5 \mathrm{nT}$ due to swaying in the ambient field. In this study, we see a deviation in the difference of around $0.5 \mathrm{nT}$ in the most disturbed cases, caused by flying up against strong winds during parts of the survey. However, P-UXO were still recovered on flight lines that were highly affected by strong winds, and we therefore expect this deviation to be due changes in sensor position within the core field, rather than due to UAV-induced noise.

If the end result of a surveying activity is a magnetic map for manual inspection, images of the raw difference may not be optimal compared to, for example, conventional data processing techniques, aimed at producing maps for manual interpretation. However, the obtained results in this study are not useless for manual interpretation. We expect this to be due to the sway-dampening towing system, as freely swaying magnetometers most likely would not have produced maps that are equivalently pleasing to the eye.

For a choice between a single airborne magnetometer with base station, and a set of dual airborne magnetometers, the added value of the scalar magnetic difference should be taken into account. Although the added value of the measured data is high, it must be weighed against the added complexity and weight of the towed system, as these parameters are crucial for successful magnetic surveying with UAVs. If the end goal is visually pleasing single-sensor scalar magnetic field data, it is also possible to combine the difference data with, for example, a diurnal correction strategy, such as a magnetic base station or tie lines, the latter resulting in very little extra cost. This may not be suitable in areas of high disturbance, such as urban areas or other places with significant anthropogenic activity.

\section{CONCLUSION}

RDI provides an alternative to conventional gradient approximations in predominantly vertically aligned dual sensor systems, and is robust to movement of the sensors, as it does not depend on a single-defined gradient alignment or direction. It simultaneously retains the independence on external variations seen in conventional finite-difference gradient surveys.

Scalar magnetic UXO survey data from two different areas have been presented, and inverted for potential targets using the point-dipole model. The point-dipole model enables the recovery of all buried UXO targets at the test facility, provided that their magnetic signatures are distinguishable. In a complex magnetic background, high order $(k \leq 2)$ differences enabled detection of UXO with minute magnetic signatures, which could not be detected from first differences using the explained approach, but this came at significant additional cost, that is, a large increase in non-UXO targets. We have shown how the differencing method can be implemented for detecting UXO through models of magnetostatic point dipoles, but the theory is easily extended to other sensor configurations and source models, given that the amount of samples of the recovered scalar magnetic differences is sufficiently high to accurately capture the modelled magnetic signatures. The combination of the first-order approximation to the measured scalar magnetic field, the two-peak initial guess method, and the point-dipole source model, enabled detection of $155 \mathrm{~mm}$ shells, as well as larger UXO, for first-order differences. Additionally, $105 \mathrm{~mm}$ projectiles and $75 \mathrm{~mm}$ canisters were detectable using higher order differences, which also showed a general increase in inversion robustness. However, 
detection of these smaller UXO required the simultaneous recovery of a substantial number of additional targets, resulting in a significantly higher cost of detection when compared to detecting the larger UXO only.

UAV-towed systems enable the collection of high-quality data in both draped-survey scenarios, as well as in coastal settings with shallow waters and surf zones, and enables target detection with the RDI approach even in cases with highly varying relative sensor positions, as seen in parts of the coastal survey that were significantly impacted by wind.

\section{ACKNOWLEDGEMENTS}

AD prepared the funding grant proposal, which resulted in this work being funded by Innovation Fund Denmark through IFD grant 615900002B. Both authors participated in the planning process and fieldwork, including data collection, and would like to thank Commander Lars Møller and the Royal Danish Navy EOD Service for their assistance in preparing the synthetic mine field. Data processing was performed by MEK, who also analysed the results with inputs from AD. The paper was written by MEK and finalized after review by AD. Both authors prepared graphics for the manuscript. We thank Eduardo Lima Simoes da Silva and the rest of the CMAGTRES group, which is led by AD, for their assistance with data collection. Ørsted (www.orsted.com) is greatly acknowledged and thanked for providing the raw data from the coastal survey. All presented data were collected using equipment from UMag Solutions (www.umagsolutions.com).

\section{REFER E N CES}

Butler, D.K., Wolfe, P.J. \& Hansen, R.O., 2001. Analytical modeling of magnetic and gravity signatures of unexploded ordnance, J. Environ. Eng. Geophys., 6(1), 33-46.

Cherkasov, S.V., Sterligov, B.V. \& Zolotaya, L.A., 2016. On the use of unmanned aerial vehicles for high-precision measurements of the earth's magnetic field, Moscow Univ. Geol. Bull., 71(4), 296-299.

Cowan, D.R., Baigent, M. \& Cowan, S., 1995. Aeromagnetic gradiometersa perspective, Explor. Geophys., 26(2-3), 241-246.

Davis, K., Li, Y. \& Nabighian, M., 2010. Automatic detection of UXO magnetic anomalies using extended Euler deconvolution, Geophysics, 75(3), G13-G20.

Finlay, C., Olsen, N., Kotsiaros, S., Gillet, N. \& Tøffner-Clausen, L., 2016. Recent geomagnetic secular variation from Swarm and ground observatories as estimated in the CHAOS-6 geomagnetic field model, Earth Planets Space, 68(1), 112, doi:10.1186/s40623-016-0486-1.

Fletcher, R., 1971. A modified Marquardt subroutine for non-linear least squares, Tech. Rep., United Kingdom Atomic Energy Authority, Theoretical Physics Division, Atomic Energy Research Establishment.

Gamey, T.J., Doll, W.E., Beard, L.P. \& Bell, D.T., 2002. Airborne vertical magnetic gradient for UXO detection, in 15th EEGS Symposium on the Application of Geophysics to Engineering and Environmental Problems, pp. cp-191, European Association of Geoscientists \& Engineers, https://doi.org/10.3997/2214-4609-pdb.191.11UXO6.

Giret, R.I., 1965. Some results of aeromagnetic surveying with a digital Cesium-vapor magnetometer, Geophysics, 30(5), 883-890.

Levenberg, K, 1944. A method for the solution of certain non-linear problems in least squares, Q. appl. Math., 2(2), 164-168.

Marquardt, D.W., 1963. An algorithm for least-squares estimation of nonlinear parameters, J. Soc. Indus. Appl. Math., 11(2), 431-441.

McFee, J.E. \& Das, Y., 1981. Determination of the parameters of a dipole by measurement of its magnetic field, IEEE Trans. Antennas Propag., 29(2), 282-287.

Misra, P. \& Enge, P., 2006, Global Positioning System: Signals, Measurements And Performance, Second Editions, Ganga-Jamuna Press, p. 206.

Nabighian, M.N., 1984. Toward a three-dimensional automatic interpretation of potential field data via generalized Hilbert transforms: fundamental relations, Geophysics, 49(6), 780-786.

Nelson, H.H. \& McDonald, J.R., 2001. Multisensor towed array detection system for UXO detection, IEEE Trans. Geosci. Remote Sens., 39(6), $1139-1146$.
Olsen, N., Hulot, G. \& Sabaka, T.J., 2014. Sources of the geomagnetic field and the modern data that enable their investigation, in Handbook of Geomathematics, pp. 227-249, Springer, doi:10.1007/978-3-642-54551-1_5.

Panofsky, W. \& Phillips, M., 1955. Classical Electricity and Magnetism, p. 15, Addison-Wesley, 2ndedn.

Parshin, A.V., Morozov, V.A., Blinov, A.V., Kosterev, A.N. \& Budyak, A.E., 2018. Low-altitude geophysical magnetic prospecting based on multirotor UAV as a promising replacement for traditional ground survey, Geo-spatial Inform. Sci., 21(1), 67-74.

Roest, W., Verhoef, J. \& Pilkington, M., 1992. Magnetic interpretation using 3-D analytic signal, Geophysics, 57, 116-125.

Salem, A., Hamada, T., Asahina, J.K. \& Ushijima, K., 2005. Detection of unexploded ordnance (UXO) using marine magnetic gradiometer data, Explor: Geophys., 36(1), 97-103.

Sanchez, V., Yaoguo, L., Nabighian, M.N. \& Wright, D.L., 2008. Numerical modeling of higher order magnetic moments in UXO discrimination, IEEE Trans. Geosci. Remote Sens., 46(9), 2568-2583.

Stoll, J.B., 2013. Unmanned aircraft systems for rapid near surface geophysical measurements, in International Archives of the Photogrammetry, Remote Sensing and Spatial Information Sciences, Bill, R. \& Grenzdorffer, G., eds, International Society for Photogrammetry and Remote Sensing. XL-1/W2, 2013UAV-g2013, pp. 391-394.

Thompson, D., 1982. EULDPH: a new technique for making computerassisted depth estimates from magnetic data, Geophysics, 47(1), $31-37$.

Tuck, L., Samson, C., Polowick, C. \& Laliberté, J., 2019. Real-time compensation of magnetic data acquired by a single-rotor unmanned aircraft system, Geophys. Prospect., 67(6), 1637-1651.

Walter, C., Braun, A. \& Fotopoulos, G., 2019. Spectral analysis of magnetometer swing in high-resolution UAV-borne aeromagnetic surveys, in 2019 IEEE Systems and Technologies for Remote Sensing Applications Through Unmanned Aerial Systems (STRATUS), pp. 1-4, doi:10.1109/stratus.2019.8713313.

Walter, C.A., Braun, A. \& Fotopoulos, G., 2019. Impact of three-dimensional attitude variations of an unmanned aerial vehicle magnetometry system on magnetic data quality, Geophys. Prospect., 67(2), 465-479.

Wang, B., Krebes, E.S. \& Ravat, D., 2008. High-precision potential-field and gradient-component transformations and derivative computations using cubic b-splines, Geophysics, 73(5), I35-I42.

Wyckoff, R., 1948. The gulf airborne magnetometer, Geophysics, 13(2), 182-208.

\section{SUPPORTING INFORMATION}

Supplementary data are available at GJI online.

Figure S1. Small UXO (UXO 1, 2 and 3) convergence results from the robustness test performed on test facility data. Bar values were obtained by stacking results from tests using 3, 5 and $7 \mathrm{~m}$ data inclusion radii. Letter codes refer to upper sensor (U), lower sensor (L) and 
double sensors (D), respectively. Numbers following each letter correspond to the differencing order (k). Bar colours represent horizontal offsets between the initial horizontal position guess and the UXO position, while individual subplots represent vertical offsets with respect to the actual UXO burial depth. Note that U1 and L1 have identical results.

Figure S2. Large UXO (UXO 4-12) convergence results from the robustness test performed on test facility data. Bar values were obtained by stacking results from tests using 3, 5 and $7 \mathrm{~m}$ data inclusion radii. Letter codes refer to upper sensor (U), lower sensor (L) and double sensors (D), respectively. Numbers following each letter correspond to the differencing order (k). Bar colours represent horizontal offsets between the initial horizontal position guess and the UXO position, while individual subplots represent vertical offsets with respect to the actual UXO burial depth. Note that $\mathrm{U} 1$ and L1 have identical results.

Figure S3. Convergence results from the robustness test performed on test facility data, using a $3 \mathrm{~m}$ data inclusion radius. Letter codes refer to upper sensor (U), lower sensor (L) and double sensors (D), respectively. Numbers following each letter correspond to the differencing order (k). Bar colours represent horizontal offsets between the initial horizontal position guess and the UXO position, while individual subplots represent vertical offsets with respect to the actual UXO burial depth. Note that U1 and L1 have identical results.

Figure S4. Convergence results from the robustness test performed on test facility data, using a $5 \mathrm{~m}$ data inclusion radius. Letter codes refer to upper sensor (U), lower sensor (L) and double sensors (D), respectively. Numbers following each letter correspond to the differencing order (k). Bar colours represent horizontal offsets between the initial horizontal position guess and the UXO position, while individual subplots represent vertical offsets with respect to the actual UXO burial depth. Note that U1 and L1 have identical results.

Figure S5. Convergence results from the robustness test performed on test facility data, using a $7 \mathrm{~m}$ data inclusion radius. Letter codes refer to upper sensor (U), lower sensor (L) and double sensors (D), respectively. Numbers following each letter correspond to the differencing order (k). Bar colours represent horizontal offsets between the initial horizontal position guess and the UXO position, while individual subplots represent vertical offsets with respect to the actual UXO burial depth. Note that U1 and L1 have identical results.

Table S1. Relative detection limits and total amount of targets recovered at the recovery threshold of each UXO at the test facility. The recovery threshold is the upper prominence limit at which a UXO can be recovered using the two-peak method. The total amount of recovered targets are shown in parenthesis. Note that apart from the buried P-UXO, three of the detected anomalies are deliberately placed scrap metal objects, and thus the listed amount contains a small bias. Since UXO 9 and 10 are always recovered as a single anomaly, we count them together as a single target.

Please note: Oxford University Press is not responsible for the content or functionality of any supporting materials supplied by the authors. Any queries (other than missing material) should be directed to the corresponding author for the paper. 